\title{
Les transferts monétaires des migrants : pays industrialisés et pays en développement
}

Yves Charbit et Isabelle Chort

\section{(2) OpenEdition}

Édition électronique

URL : https://journals.openedition.org/remi/2825

DOI : 10.4000/remi.2825

ISSN : $1777-5418$

Éditeur

Université de Poitiers

\section{Édition imprimée}

Date de publication : 1 juillet 2006

Pagination : 127-154

ISBN : 2-911627-42-3

ISSN : 0765-0752

Référence électronique

Yves Charbit et Isabelle Chort, "Les transferts monétaires des migrants : pays industrialisés et pays en développement », Revue européenne des migrations internationales [En ligne], vol. 22 - n² 2 2006, mis en ligne le 01 juin 2009, consulté le 14 avril 2022. URL : http://journals.openedition.org/remi/2825 ; DOI : https://doi.org/10.4000/remi.2825

Ce document a été généré automatiquement le 14 avril 2022.

(c) Université de Poitiers 


\title{
Les transferts monétaires des migrants : pays industrialisés et pays en développement
}

\author{
Yves Charbit et Isabelle Chort
}

1 Dans une précédente étude (Charbit, 2006) nous avions proposé un bilan des transferts monétaires ou en nature, que nous avions analysés dans la perspective de leur contribution au développement des pays d'origine des travailleurs migrants résidant à l'étranger. ${ }^{1}$ L'on avait indiqué quels facteurs sociologiques, institutionnels et politiques étaient susceptibles d'expliquer le recours à l'une ou l'autre forme de flux. Pour les transferts officiels, les données retenues étaient celles de 2002. La comparaison avec certains agrégats (PIB, population non migrante) conduisait à un résultat qui mérite d'être rappelé brièvement, car il apportait un élément de réponse à la question centrale de savoir si les transferts contribuent au développement. Un calcul de corrélation brut entre les transferts par habitant et le PIB par habitant pour 57 pays révélait une corrélation certes positive, mais faible $(+0,32)$. Ce résultat qui semble aller à contrecourant du bon sens est somme toute prévisible et en un certain sens souhaitable du point de vue du développement. Résultat prévisible parce que les migrants à l'étranger ne représentent qu'une faible fraction de la population active et donc du potentiel de création de richesse : les transferts jouent donc un rôle marginal dans le PIB. Ce constat est cohérent avec certaines études de cas. En Corée par exemple, entre 1976 et 1981 les transferts n'expliquent que 3 à 7 \% de la croissance du PIB (Kim, 1983 cité par Arnold, 1992). Il est par ailleurs souhaitable dans la perspective du développement que la migration ne soit pas un facteur majeur de contribution au PIB, car ce serait le signe d'une forte dépendance à l'égard des pays demandeurs de travailleurs migrants et donc d'une cause supplémentaire de fragilité économique des pays d'émigration. Mais la corrélation évoquée ci-dessus peut aussi se lire en sens inverse : la richesse d'un pays (mesurée par son PIB par habitant) déterminerait alors le montant de transferts qu'il reçoit, via, par exemple, l'effet du revenu des populations sur leurs comportements migratoires, mais aussi sur leurs caractéristiques socioprofessionnelles. Aussi il ne faut 
pas confondre corrélation et relation de causalité ni tirer trop de conclusions d'un tel constat statistique brut.

Cet article, qui est centré sur les seuls transferts monétaires officiels, propose de nouvelles ouvertures. D'abord, un échantillon de 103 pays a été retenu. En outre la comparaison de deux séries de données (1985 et 2003) permet de constater les permanences ou au contraire les changements survenus sur près d'une décennie. Enfin de nouveaux indicateurs ont été ajoutés à ceux antérieurement utilisés; ils correspondent à une problématique légèrement différente, plus macro-économique, puisque les flux sont comparés à d'autres postes de la balance des paiements des pays en développement.

3 Alors que jusqu'à la fin des années 1990, les transferts des migrants en direction des pays en développement étaient relativement peu documentés par rapport aux deux autres flux, sur lesquels se focalisait l'attention, les investissements directs étrangers (IDE) et l'aide publique au développement (APD), ils sont aujourd'hui reconnus comme un enjeu de taille pour le développement. Notamment sous la pression des institutions de Bretton Woods, et afin de disposer de données internationales fiables et homogènes, plusieurs initiatives sont en cours pour améliorer la mesure des transferts. C'est ainsi qu'un groupe de travail sur l'amélioration du traitement statistique des transferts, présidé par la Banque Mondiale a été mis en place à la suite de la recommandation du G 8 lors du sommet de Sea Island en 2004. Il a pour objectif à l'horizon 2006 l'amélioration des données et la clarification de la définition des transferts. Outre le groupe de travail conduit par les Nations Unies ${ }^{2}$, on peut également citer sur ce même thème le travail en cours de la Banque Mondiale en collaboration avec le DFID (Inter-Agency Remittances Task Force).

4 En 2004, selon la Banque Mondiale, plus de 180 millions de personnes vivent hors de leur pays d'origine. Étroitement lié au phénomène migratoire, le volume des fonds rapatriés par les travailleurs migrants a doublé en dix ans, atteignant en 2004, toujours selon la Banque Mondiale, le montant de 216 milliards de dollars EU. 151 milliards de dollars EU, soit plus de deux tiers de ces fonds, arrivent dans des pays en développement. Pour certains de ces pays les transferts des émigrés sont la première source de devises, ce qui témoigne de l'importance de ces apports. Ils tendent par ailleurs à dépasser l'aide au développement, devenant ainsi le second flux financier à destination des pays en développement derrière les investissements directs étrangers (International Technical Meeting on Measuring Migrant Remittances, World Bank, Hussein, 2004). Les transferts font donc l'objet d'une attention particulière en tant que source de financement externe pour les pays en développement, et qui plus est, source relativement stable (Solimano, 2003 ; Sander et Maimbo, 2003). Cette stabilité constitue la particularité de ce flux, à la différence notamment des investissements directs étrangers (IDE) réputés pour être particulièrement volatils dans les pays en développement.

5 Après avoir brièvement évoqué quelques problèmes de mesure (1), on s'attachera d'abord aux pays « émetteurs » de transferts migratoires et plus précisément à ceux qui affichent les montants les plus élevés de transferts sortants dans leur balance des paiements (2) On évoque souvent les pays du Golfe comme l'exemple archétypal d'économies employant une main-d'œuvre étrangère nombreuse et génératrice de transferts importants. Nous chercherons à répondre à deux questions : les volumes des transferts dans ces pays confirment-ils l'opinion couramment répandue sur l'ampleur 
des transferts générés par la manne pétrolière ? Cette question en appelle une autre : leur type d'économie induit-il une spécificité éventuelle? Qu'en est-il dans les économies industrielles plus diversifiées? Nous examinerons le cas de la France, sur laquelle se conclura cette partie. Nous examinerons ensuite la situation des pays en développement qui reçoivent les transferts de leurs travailleurs à l'étranger (3), nous prendrons à titre d'exemple les données relatives à deux années, 1985 et 2003. On constatera que la comparaison des principales destinations des flux à près de vingt ans d'intervalle, met en évidence une relative stabilité, du moins dans la hiérarchie des plus gros destinataires. Est-ce à dire que la migration ne constituerait pas un état transitoire dans le développement d'un pays mais une sorte de spécialisation? Ce point soulève la question de la dépendance des pays à l'égard des flux de transferts, que nous examinerons enfin dans la quatrième partie en comparant ces flux à quatre indicateurs: le produit intérieur brut, les revenus du tourisme, les exportations, les investissements directs étrangers (IV).

\section{Problèmes de mesure des transferts}

Les transferts informels ont été exclus de l'analyse présentée dans cet article, qui compare les transferts officiels à deux autres flux assez bien enregistrés, et qui sont eux aussi lisibles sur la balance des paiements, à savoir les investissements directs étrangers (IDE) et l'aide publique au développement (APD). La composante informelle des transferts échappe à tout enregistrement et ne peut être mesurée de façon aussi précise que la composante "officielle»; elle est tout au mieux évaluée par des estimations à partir d'enquêtes auprès des ménages ou d'enquêtes sur les migrants aux postes de frontière. C'est le cas en équateur ou au Honduras (Luna Martinez, 2005). Toutefois rares sont les pays qui mettent en œuvre de telles enquêtes, et d'un pays à l'autre la méthodologie employée varie, rendant délicate la comparaison. Cette composante informelle des transferts, qui existe dans tous les pays et qui est loin d'être négligeable, conduit donc à une sous-estimation systématique des flux de transferts ${ }^{3}$.

De plus, la mesure des transferts qui passent par les canaux formels n'est, elle non plus, totalement satisfaisante (Brown, 1994). En effet de nouvelles difficultés surgissent. En premier lieu la définition du "migrant», qui est au cœur de la mesure des transferts, fait l'objet des discussions des groupes de travail précédemment évoqués. N'est considéré comme migrant selon le FMI que l'individu absent depuis plus d'un an de son pays d'origine. Cette limite arbitraire d'un an produit une définition restrictive à l'excès puisqu'elle n'inclut ni les transferts liés aux migrations saisonnières ni les transferts des migrants "de long terme " effectués au cours de leur première année d'installation à l'étranger. Il serait souhaitable d'introduire dans les statistiques les données concernant une catégorie de migrants de «court terme: $»^{4}$ qui désigne les personnes migrant pour une période de 3 mois à $1 \mathrm{an}^{5}$. En effet la pratique des migrations saisonnières est fréquente et génère pour ce type de migrants des revenus substantiels dont une part parfois importante (Mboup, 2000) est transférée dans le pays d'origine. Pour ne citer qu'un exemple, on peut évoquer le cas des travailleurs marocains, ouvriers saisonniers agricoles dans le sud de la France, dont la capacité d'épargne se monte à près de $80 \%$ de leur revenu (Charef, 2006).

D'autres erreurs concernant les transferts officiels sont dues au mauvais enregistrement des transactions dans la balance des paiements. Tel est le cas en 
particulier des transferts en nature, enregistrés comme tels mais agrégés au poste "Erreurs et Omissions », ou encore les opérations réalisées sur les comptes bancaires en devises détenus par les résidents qui apparaissent dans les comptes des nonrésidents. Ces deux erreurs parmi les plus fréquentes conduisent à sous-estimer les transferts effectués.

Pour chaque pays, trois indicateurs ont été retenus : les valeurs absolues (en millions de dollars), les transferts rapportés au PIB (en pourcentage du PIB), les transferts rapportés à l'effectif de la population des pays d'origine des travailleurs (exprimés en dollars par habitant). Selon l'indicateur choisi, le classement des pays est susceptible de changer. Les cartes de la troisième partie illustrent la répartition mondiale des transferts selon chacun de ces trois indicateurs. Les données ayant permis la construction de ces figures sont annexées à l'article (tableaux 5 à 11). On trouvera en particulier (tableau 5) le classement en fonction des transferts reçus en 2003 des pays en développement pour lesquels nous disposons de données à cette date, soit 103 pays. Dans ce tableau figurent en outre les valeurs prises par les deux autres mesures des transferts que nous utilisons (transferts en pourcentage du PIB, transferts en dollars par habitant). Ces données sont mises en regard des valeurs enregistrées en 1985, afin d'élargir la réflexion en introduisant une analyse longitudinale.

La définition internationale des transferts officiels qui est retenue ici est celle proposée par le FMI (Balance of Payments Manual, $5^{\mathrm{e}}$ édition, 1993). Bien que critiquable elle a le mérite, étant diffusée auprès de toutes les instances nationales, d'homogénéiser la production statistique sur les transferts migratoires et de faciliter les comparaisons internationales. Les transferts des migrants, selon le FMI résultent de l'agrégation de trois postes de la balance des paiements: les "Compensations des employés", les «Transferts des travailleurs » et les « Autres transferts ». Le schéma ci-dessous (figure 1) montre les "pertes" subies par les données à différents niveaux. Les données macroéconomiques sur les transferts que nous utilisons, résultant des définitions du FMI, ne rendent compte que d'une part des transferts totaux, non nécessairement la plus importante.

Fig. 1. Transferts migratoires et mesure des transferts

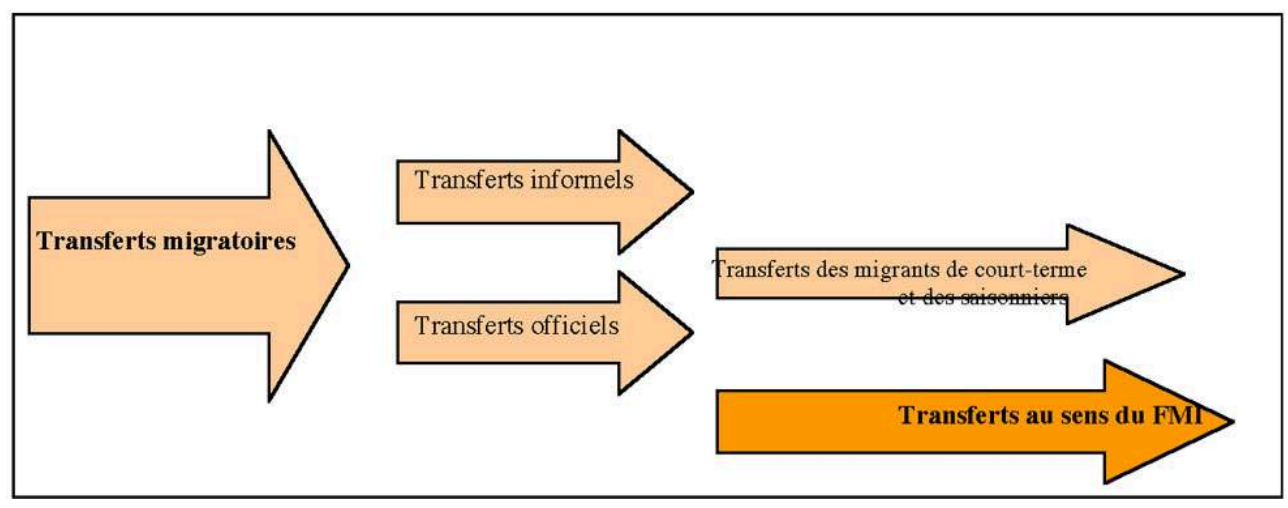

\section{Les pays émetteurs de transferts}

11 Parmi les principaux émetteurs de transferts migratoires en 2003, on peut distinguer (tableau 1) deux sous-groupes de pays: le premier, qui est constitué d'économies 
développées avec en tête les états-Unis, les principaux pays de l'Union Européenne dont la France, le Japon, Israël, l'Australie ; le second rassemble des pays producteurs de pétrole : Arabie Saoudite, Koweït et Oman. L'Arabie Saoudite occupe la deuxième place du classement en 2003 avec un volume de transferts sortants égal à quatre fois celui émis par la France.

\section{Spécificité des pays pétroliers}

Les transferts obéissent-ils à la même logique dans ces deux types d'économie ? Est-il légitime de postuler une spécificité des pays du golfe persique en matière de rapatriement des fonds en provenance de la main-d'œuvre immigrée ? On peut en effet considérer que du fait de la quasi-spécialisation de ces pays dans le secteur pétrolier et des conditions de vie des étrangers dans ces pays, les opportunités de dépense et a fortiori d'investissement pour les travailleurs étrangers sous contrat sont moins nombreuses que dans les économies développées et diversifiées du premier sousgroupe. Ils seraient donc contraints à une forme d'épargne forcée. Pour tenter de répondre à cette question, le tableau 2 met en parallèle quelques pays choisis dans ces deux sous-groupes.

Tableau 1 : Les 20 premiers pays émetteurs de transferts migratoires en 1985 et en 2003

\begin{tabular}{|c|c|c|c|}
\hline \multicolumn{2}{|c|}{2003} & \multicolumn{2}{|c|}{1985} \\
\hline Pays & $\begin{array}{c}\text { Transferts sortants } \\
\text { (en millions de dollars) }\end{array}$ & Pays & $\begin{array}{c}\text { Transferts sortants } \\
\text { (en millions de dollars) }\end{array}$ \\
\hline Etats-Unis & 34075 & Etats-Unis & 6330 \\
\hline Arabie saoudite & 14916 & Arabie saoudite & 5199 \\
\hline Allemagne & 9852 & France & 4173 \\
\hline Suisse (2002) & 9222 & Allemagne & 3598 \\
\hline France & 4718 & Suisse & 2509 \\
\hline Luxembourg & 4632 & Koweït & 1044 \\
\hline Italie & 4367 & Oman & 950 \\
\hline Espagne & 4009 & $\begin{array}{l}\text { Jamahiriya } \\
\text { arabe libyenne }\end{array}$ & 859 \\
\hline Malaisie (2002) & 3826 & $\begin{array}{l}\text { Belgique- } \\
\text { Luxembourg }\end{array}$ & 779 \\
\hline Fédération de Russie & 3233 & Bahureïn & 778 \\
\hline Royaume-Uni & 2624 & Afrique du Sud & 722 \\
\hline Pays-Bas & 2349 & Pays-Bas & 658 \\
\hline Israēl & 2314 & Italie & 606 \\
\hline Belgique & 2273 & Israël & 410 \\
\hline Koweït & 2144 & Australie & 312 \\
\hline Japon & 1773 & Nigéria & 298 \\
\hline Oman (2001) & 1532 & Suède & 290 \\
\hline Australie & 1495 & Côte d'Ivoire & 287 \\
\hline Autriche & 1473 & Jordanie & 236 \\
\hline $\begin{array}{l}\text { Corée, République de } \\
\text { (2002) }\end{array}$ & 1398 & Venezuela & 217 \\
\hline
\end{tabular}

Source : Manuel de Statistiques de la CNUCED, 2005. 
Tableau 2 : Transferts sortants de quelques économies développées et de pays pétroliers

\begin{tabular}{|l|c|c|c|}
\hline 2003 & Transferts en \% du PIB & $\begin{array}{c}\text { Etrangers en \% de la } \\
\text { population totale }\end{array}$ & $\begin{array}{c}\text { Transferts en S US par } \\
\text { étranger }\end{array}$ \\
\hline Arabie Saoudite & 7,19 & 21,70 & 2838 \\
\hline Koweït & 6,23 & Non disponible & Non disponible \\
\hline Oman & 7,68 & Non disponible & Non disponible \\
\hline France & 0,27 & 4,58 & 1713 \\
\hline États-Unis & 0,31 & 6,31 & 1835 \\
\hline Allemagne & 0,41 & 19,00 & 629 \\
\hline Royaume-Uni & 0,15 & 8,21 & 539 \\
\hline Luxembourg & 17,82 & 27,59 & 37047 \\
\hline
\end{tabular}

Sources : Manuel de Statistiques de la CNUCED, 2005 et OCDE (pour le nombre d'étrangers) pays pétroliers que dans les économies développées, à l'exception notable du Luxembourg. Le Luxembourg fait beaucoup appel depuis plusieurs décennies à la maind'œuvre étrangère, mais c'est d'une main-d'œuvre essentiellement qualifiée et en grande majorité européenne qu'il s'agit: un tiers des étrangers présents au Luxembourg sont en effet originaires de pays voisins ou proches (Belgique, France, Allemagne, Pays-Bas).

Toutefois la comparaison du poids des transferts sortants dans le PIB entre les deux sous-groupes de pays n'a de valeur que mise en regard de la part des migrants dans la population du pays. En effet, un pays faisant massivement appel à la main-d'œuvre immigrée va distribuer une part plus importante du PIB en salaires aux migrants, ce qui va, toutes choses égales par ailleurs, mécaniquement gonfler les montants transférés. Aussi serait-il intéressant de comparer les montants transférés par migrant dans les deux sous-groupes de pays. Malheureusement, nous n'avons de données sur le nombre d'étrangers que pour l'Arabie Saoudite et en outre elles datent de 2000. Encore une fois, les chiffres sont difficiles à interpréter : d'une part le nombre d'étrangers résidant dans le pays n'est pas nécessairement égal au nombre d'actifs (parmi les étrangers peuvent se trouver des retraités, des enfants, etc.), et d'autre part les définitions ne sont pas les mêmes dans tous les pays. Ainsi en Allemagne, les personnes de nationalité allemande (de naissance ou par acquisition ultérieure) nées à l'étranger ne sont pas distinguées des personnes étrangères nées à l'étranger. Aussi approcher le nombre de migrants par le nombre «d'étrangers " mentionné dans le tableau conduit à surestimer de manière importante la part des «migrants » dans la population allemande. Il en est de même pour la Royaume-Uni. Enfin, de manière plus générale, les migrations clandestines étant impossibles à chiffrer, les données officielles sous-estiment généralement les effectifs migratoires.

Une fois émises toutes ces réserves, le montant transféré par « migrant » est nettement plus important en Arabie Saoudite que dans les cinq économies développées qui figurent au tableau 2, le Luxembourg constituant une exception. Ce constat tendrait à confirmer l'hypothèse de spécificité des pays pétroliers. 


\section{Le cas de la France}

16 La France peut être prise pour illustrer les logiques des économies diversifiées. Nous n'avons pas de données permettant de désagréger les transferts sortants en fonction du pays de destination, mais on peut penser que la corrélation est forte en Europe entre les transferts émis par un pays $A$ donné à destination d'un pays $B$ et le nombre de ressortissants du pays B présents sur le territoire de A.

17 La France est le cinquième pays émetteur de transferts migratoires en 2003, avec 4,7 milliards de dollars. Elle était troisième en 1985, derrière les États-Unis et l'Arabie Saoudite. On le sait, les populations étrangères présentes en France viennent majoritairement de l'espace méditerranéen : Europe du Sud (Portugal, Italie, Espagne), Turquie, Maghreb (Algérie, Maroc, Tunisie). En l'absence de données sur la destination des transferts migratoires sortant de France on peut raisonnablement estimer que les flux de transferts sont dirigés vers les pays d'origine des principales populations étrangères présentes sur le territoire français, sous réserve de la sous-estimation des populations étrangères liées à l'immigration clandestine ou à des caractéristiques propres à tel ou tel pays. Ainsi le cas des trois pays du Maghreb (tableau 3) illustre bien le risque que les montants des transferts officiels ne correspondent pas à la réalité des flux.

Tableau 3 : Transferts migratoires officiels depuis la France vers le Maghreb, 1993-2002

\begin{tabular}{|l|l|l|l|}
\hline Année & Algérie & Maroc & Tunisie \\
\hline 1993 & 4,2 & 973,0 & 81,2 \\
\hline 2002 & $300^{*}$ & 3300 & 1000 \\
\hline
\end{tabular}

Sources : 1993 : Lebon, 1994

2002, Tunisie et Maroc : Manuel de Statistiques de la CNUCED, 2005.

2002, Algérie : estimation. Les données n'étant pas disponibles dans le Manuel de Statistiques de la CNUCED, ce pays ne figure pas non plus dans le tableau 5 en annexe.

Les chiffres de 1993 avaient suscité les commentaires suivants: "Vers l'Algérie, d'autres pratiques telles que l'apport de biens, de marchandises, de billets de banque français ou l'existence de systèmes de compensation ont, depuis longtemps, remplacé les transferts par les canaux bancaires ou postaux " (Lebon, 1994: 56). Ils restent pertinents pour 2001. Safir (1996:155), citant Lebon, concluait à juste titre: « l'émigration algérienne ne transfère plus que des sommes très faibles, complètement atypiques, sans aucun rapport avec le potentiel financier qu'elle représente $»^{6}$.

\section{Les pays destinataires}

\section{Les transferts en 1985 et 2003}

19 La question des transferts migratoires ne renvoie pas aux mêmes enjeux dans le cas des pays en développement et des pays développés. Leur poids dans le PIB est d'ailleurs très différent : ainsi les transferts entrants en France en 2003 ne représentent que 0,65\% de son $\mathrm{PIB}^{7}$. Les données présentées ici sur les transferts entrants, qui proviennent du 
Manuel de Statistiques de la CNUCED, concernent les « pays en développement : » ainsi que les pays de la CEI et les pays d'Europe du Sud non-membres de l'Union Européenne et non répertoriés dans la catégorie « autres pays développés d'Europe ». migratoires à deux dates successives, le tableau 4 indique les 10 premiers pays destinataires de transferts (en millions de \$ US) en 2003 et en 1985, première et la dernière année pour lesquelles le Manuel de Statistiques de la CNUCED présente des données relatives aux transferts des migrants.

Tableau 4 : Les 10 premiers pays en développement destinataires de transferts en 1985 et en 2003

\begin{tabular}{|c|c|c|c|}
\hline \multicolumn{2}{|c|}{2003} & \multicolumn{2}{|c|}{1985} \\
\hline Rang & $\begin{array}{c}\text { Transferts } \\
\text { en millions de } \$\end{array}$ & Rang & $\begin{array}{c}\text { Transferts } \\
\text { en millions de \$ }\end{array}$ \\
\hline 1 Mexique & 14594,7 & 1 Egypte & 3211,7 \\
\hline 2 Inde (2002) & 8410,9 & $\begin{array}{l}2 \text { Yougoslavie } \\
\text { (anc. RFS) }\end{array}$ & 3106,0 \\
\hline 3 Philippines & 7880,0 & 3 Pakistan & 2537,1 \\
\hline 4 Pakistan & 3964,0 & 4 Inde & 2469,2 \\
\hline 5 Maroc & 3627,8 & 5 Turquie & 1714,0 \\
\hline 6 Colombie & 3075,9 & 6 Mexique & 1616,0 \\
\hline 7 Egypte & 2960,9 & 7 Jordanie & 1022,2 \\
\hline 8 Bangladesh (2002) & 2858,1 & 8 Maroc & 972,5 \\
\hline 9 Brésil & 2821,3 & 9 Thaïlande & 877,3 \\
\hline 10 Chine (2002) & 2353,1 & 10 Philippines & 806,0 \\
\hline
\end{tabular}

Source : Manuel de Statistiques de la CNUCED, 2005.

Quatre tendances se dégagent de ce tableau:

- la croissance des flux de transferts en direction des pays en développement, qui ne s'expliquent que partiellement par l'inflation survenue entre ces deux dates.

- la concentration des flux monétaires : en 2003 la somme des transferts en direction des dix plus gros destinataires se monte à 52,5 milliards de dollars, soit près de $61 \%$ des flux destinés à notre échantillon de 104 pays (voir tableau 5 en annexe). Les quarante plus gros destinataires reçoivent quant à eux 82,7 milliards de dollars soit plus de $95 \%$ du total. En 1985, les huit plus gros destinataires (constituant le premier décile de notre échantillon qui ne comprend que 80 pays) recevaient $68 \%$ du total des transferts (soit un montant de 16,6 milliards de dollars). Doit-on conclure à une concentration moindre des flux en 2003 ? La comparaison des concentrations en 1985 et 2003 nécessite d'être prudent : nous n'avons en 
1985 que quatre-vingt pays dans notre échantillon et il manque en particulier un certain nombre de pays d'Amérique du Sud (Équateur, Pérou, Nicaragua... ${ }^{10}$. Or ces pays font en 2003 partie des gros destinataires de transferts migratoires sans entrer toutefois dans le premier décile. Il se peut que leur absence biaise le calcul de concentration à la hausse, si l'on suppose que leur place dans le classement était comparable en 1985 à ce qu'elle est en 2003. En dépit de ces réserves, le fait majeur, en 2003 comme en 1985, est la forte concentration des flux de transferts migratoires.

- la relative permanence du classement : les flux de transferts migratoires ont pour l'essentiel les mêmes destinations en 1985 et en 2003. Parmi les dix premiers pays destinataires en 1985, six sont encore parmi les dix premiers destinataires en 2003, et deux (la Jordanie et la Thaïlande) sont respectivement $12^{\mathrm{e}}$ et $14^{\mathrm{e}}$. Nous n'avons pas de point de comparaison pour la Yougoslavie, classée à la seconde place en 1985 et qui a éclaté en plusieurs États depuis. Enfin la Turquie fait figure d'exception : dans notre tableau elle passe de la $5^{\mathrm{e}}$ place à la $27^{\mathrm{e}}$ place de 1985 à 2003. Ce « recul » apparent est très probablement imputable à une erreur dans les données du Manuel de Statistiques de la CNUCED (2005) : en effet en 2002 la Turquie recevait encore 1936 millions de dollars de transferts (Manuel de Statistiques de la CNUCED, 2005). Par ailleurs, selon Içduygu (2005) les transferts entrants pour la Turquie en 2003 seraient de l'ordre de 2000 millions de dollars, ce qui la placerait au $14^{\text {ème }}$ rang des pays récepteurs en 2003.

- Des nouveaux venus dans le groupe des plus gros destinataires en 2003 : le Bangladesh (passé de la $13^{\mathrm{e}}$ place à la $8^{\mathrm{e}}$ ), la Chine (de $18^{\mathrm{e}}$ à $\left.10^{\mathrm{e}}\right)$, la Colombie (de $26^{\mathrm{e}}$ à $6^{\mathrm{e}}$ ) et le Brésil (de $36^{\mathrm{e}}$ à $\left.9^{\mathrm{e}}\right)$.

Trois cartes visualisent la répartition géographique des transferts : sur la première (fig. 2 ci-dessus) figurent le volume des transferts reçus, exprimé en millions de dollars ; la deuxième (fig. 3) est construite à partir des transferts en pourcentage du PIB, la troisième (fig. 4) à partir des transferts en US \$ par habitant.

Fig. 2. Transferts migratoires officiels dans le monde en développement, 2003 (en millions de dollars)

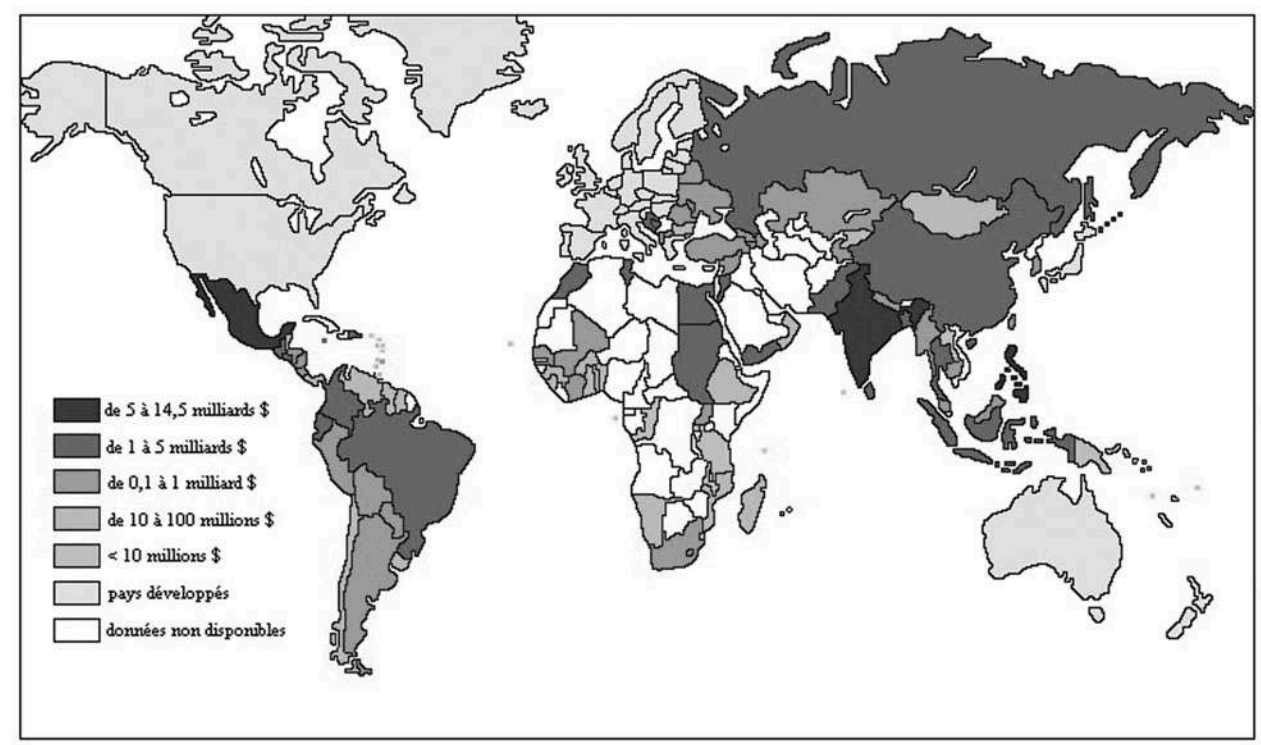


Fig. 3. Transferts migratoires officiels dans le monde en développement, 2003 (en dollars par habitant et par an)

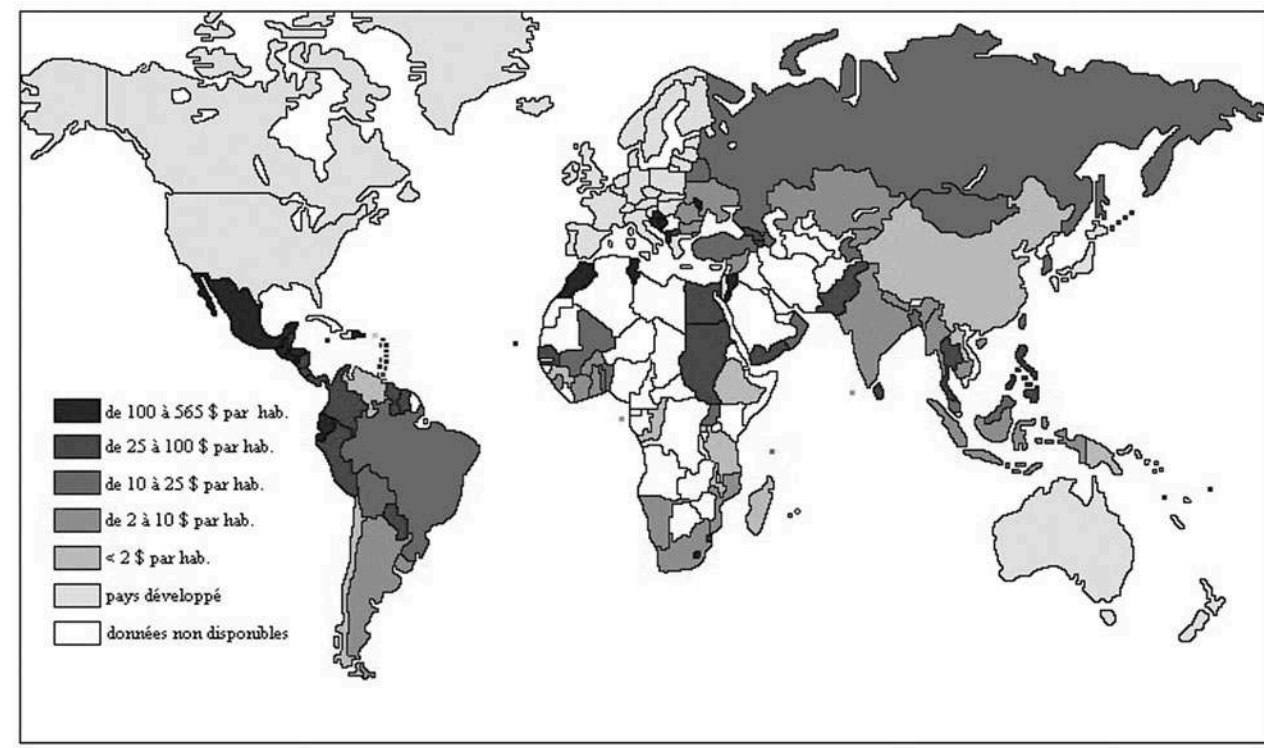

Fig. 4. Transferts migratoires officiels dans le monde en développement, 2003 (en pourcentage du PIB)

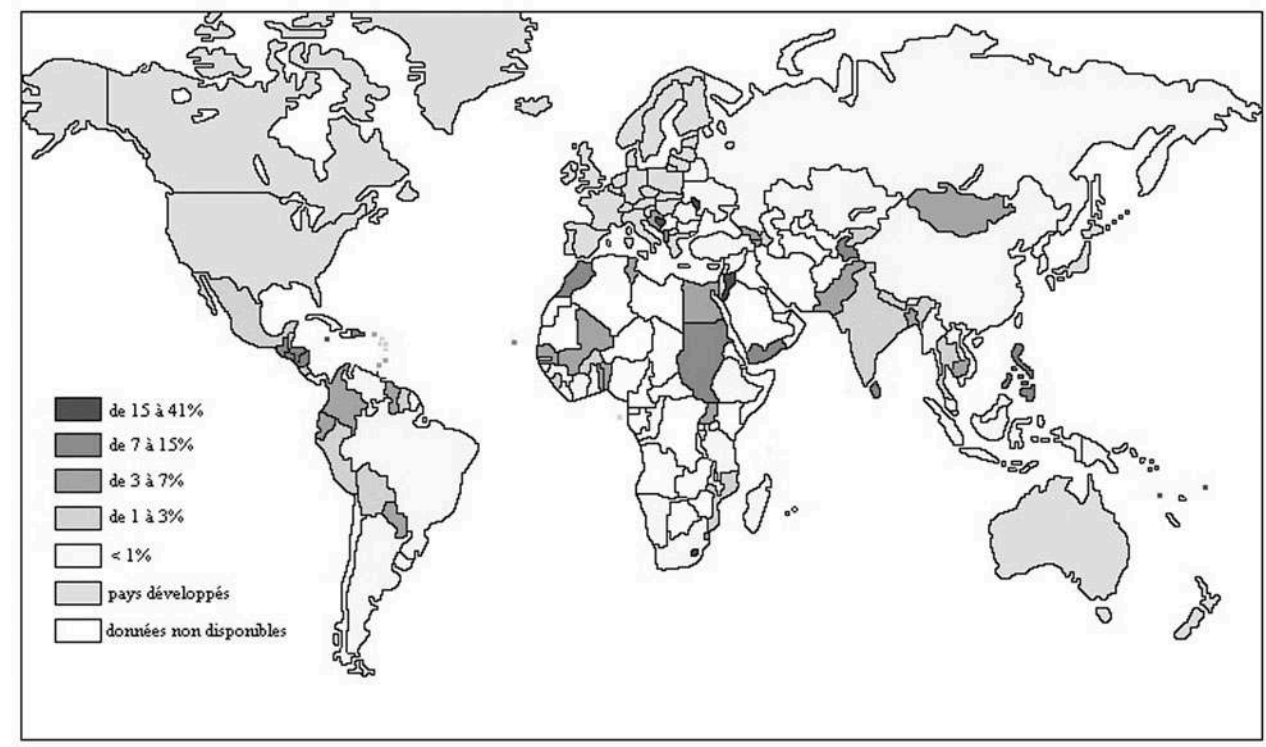

Les trois figures ne sont pas superposables: l'appréhension de l'importance des transferts est fonction de l'indicateur retenu pour les mesurer. Il est toutefois difficile de sélectionner "le meilleur indicateur", car celui-ci dépend de ce que l'on veut mesurer : l'indicateur de volume (millions de dollars) donne une idée de la destination des flux financiers (vision financière). Les transferts rapportés au PIB fournissent une image de la dépendance des économies à l'égard de cette source de revenu (vision «macroéconomique»). Enfin, l'indicateur des transferts en dollar par habitant, en normalisant par la population permet de relativiser l'importance des transferts pour un pays donné. Il est le préalable à une analyse plus fine (que nous ne mènerons pas ici) de la réponse à la pauvreté que constitue la «manne » des transferts pour les populations 
des pays d'origine des travailleurs. Cependant les écarts très profonds que l'on constate entre les pays pour ce dernier indicateur (tableau 5 en annexe) conduisent à souligner trois facteurs qui jouent de manière divergente :

- si les migrants touchent de faibles revenus parce qu'ils travaillent dans des pays où le niveau moyen de rémunération de la main-d'œuvre est faible (par exemple dans le secteur minier en Afrique du sud par rapport aux salaires versés en France dans le BTP), les transferts vont être plus faibles ;

- si le pays d'origine où arrivent ces transferts compte peu d'habitants, et que les travailleurs à l'étranger sont nombreux, les transferts par tête vont au contraire être très élevés ;

- enfin et comme on l'a évoqué plus haut, si les migrants ne peuvent rien dépenser dans certains pays (Golfe notamment), ils sont contraints à une épargne forcée et vont transférer plus d'argent.

La première des cartes (fig. 2) illustre la destination des flux de transferts : on y repère les principaux pays destinataires présentés dans le tableau 4, mais en élargissant le champ à tous les pays destinataires on remarque également la grande hétérogénéité des montants reçus. L'absence de statistiques pour la plupart des pays d'Afrique apparait en creux sur cette carte.

La seconde carte (fig. 3) met en évidence la dépendance des économies à l'égard de cette source de revenu que constituent les transferts migratoires entrants en les comparant à la richesse nationale (l'ensemble des revenus du pays). Il est toutefois ambigu de parler de dépendance, le terme étant connoté négativement, et ceci pour trois raisons : nous avons noté plus haut la stabilité constatée des flux de transferts des migrants, même s'il y a basculement dans le secteur informel, qui s'oppose à l'instabilité du flux des investissements directs étrangers, très sensibles aux aléas politiques. Enfin, il n'est pas nécessairement plus malsain pour une économie de dépendre pour son financement des transferts des migrants plutôt que de l'aide publique au développement par exemple. La question qui se pose en revanche, c'est celle de la spécialisation de fait des économies nationales dans le "secteur d'activité " de la migration, lorsque le pays n'a pas d'autres sources de revenus que les transferts. Si elle existe, quels sont les enjeux de la pérennisation d'une solution supposée transitoire par rapport aux différentiels démographiques et de développement entre pays d'émigration et pays d'accueil?

La dernière carte (fig. 4) met en évidence une caractéristique géographique de la répartition des transferts, celle de la logique des migrations frontalières. Les transferts par habitant sont les plus élevés en bordure des régions développées, au niveau de vie par conséquent élevé et demandeuses de main-d'œuvre. Le Mexique, les pays d'Amérique centrale et les Iles des Caraïbes bénéficient ainsi de leur proximité avec les États-Unis. Le Maroc, la Tunisie, l'Albanie ou les pays de l'ex-Yougoslavie bénéficient de leur position sur le pourtour de l'Union Européenne.

\section{Analyse par grandes régions}

La comparaison des trois cartes révélait une grande hétérogénéité des situations relativement aux transferts migratoires au sein du monde en développement. De nouveaux indicateurs vont cette fois nous permettre de mettre en évidence l'hétérogénéité intra-régionale des situations des différents pays relative à la question des transferts. L'indicateur des transferts en pourcentage des revenus du tourisme 
permet d'évaluer l'importance respective des deux principales sources de devises. Ceux des transferts en pourcentage des investissements directs étrangers (IDE) et les transferts en pourcentage des exportations donnent une mesure de la structure du financement des économies. Dans les limites de ce bref bilan, il est bien évidemment impossible de fournir pour chaque cas particulier que les indicateurs révéleront, une explication appropriée.

Dans cinq pays d'Afrique (Lesotho, Cap-Vert, Sénégal, Burkina Faso et Sierra Leone) les transferts des migrants sont cinq à sept fois plus élevés que les investissements directs étrangers (figure 5).

Fig. 5. Afrique : transferts migratoires, investissements étrangers, exportations et tourisme, 2003

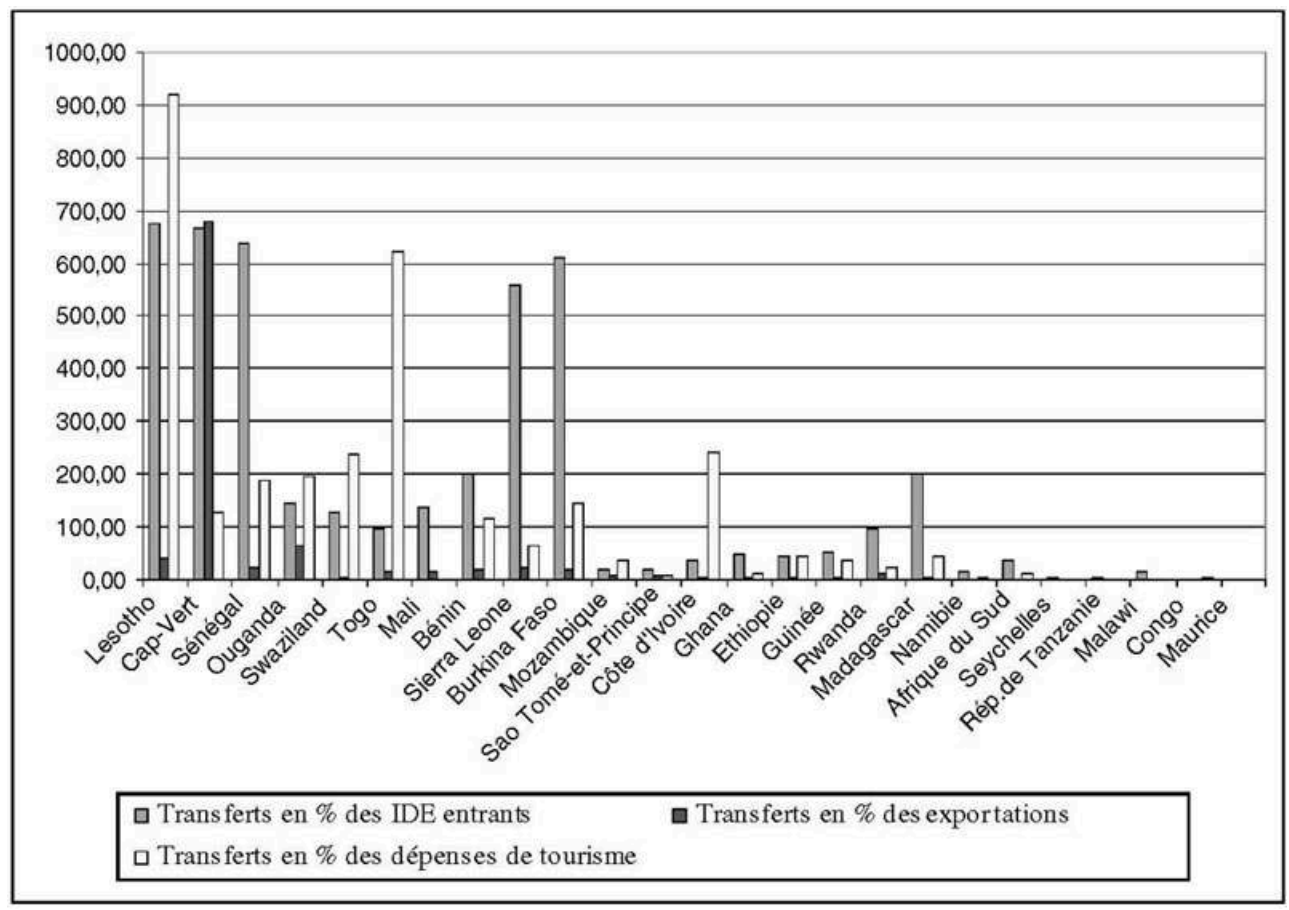


Fig. 6. Amérique latine : transferts migratoires, investissements étrangers, exportations et tourisme, 2003

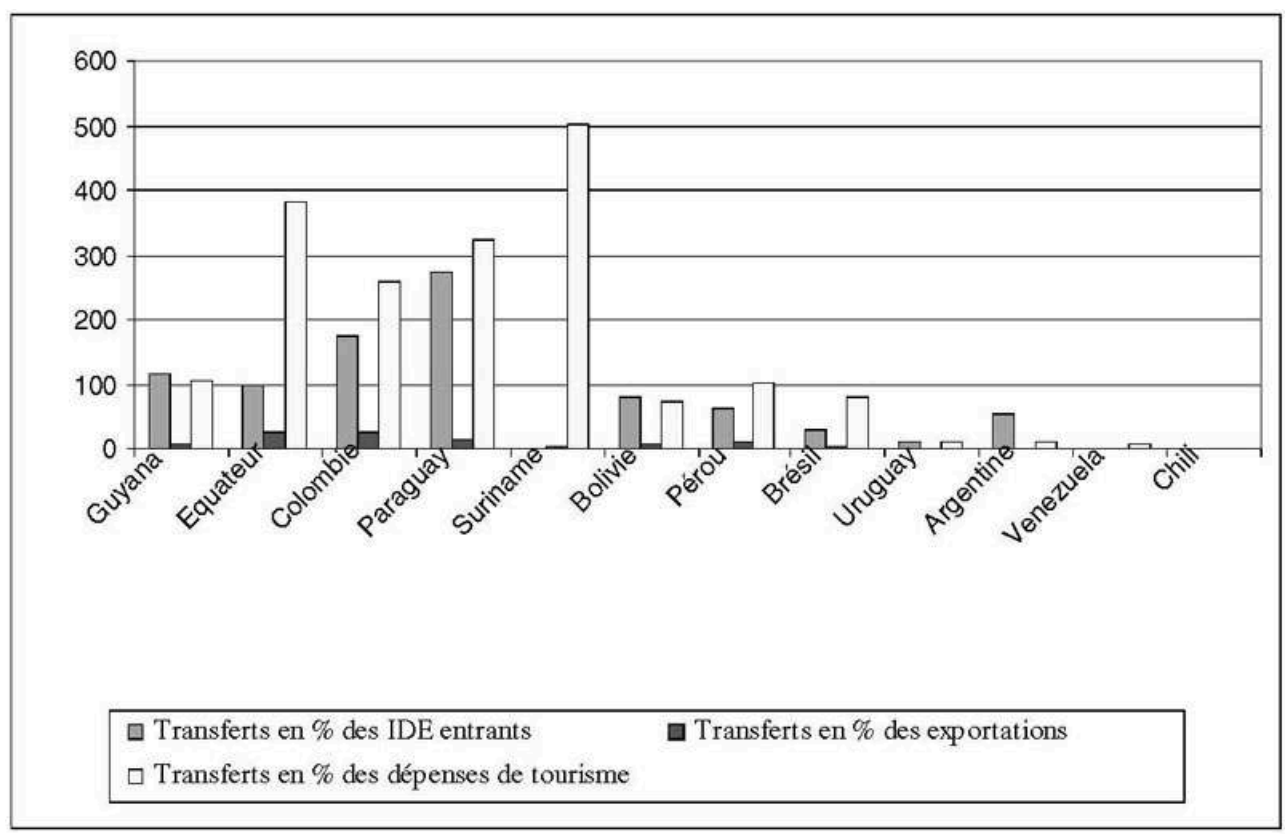

Fig.7. Amérique centrale et Caraïbes : transferts migratoires, investissements étrangers, exportations et tourisme, 2003

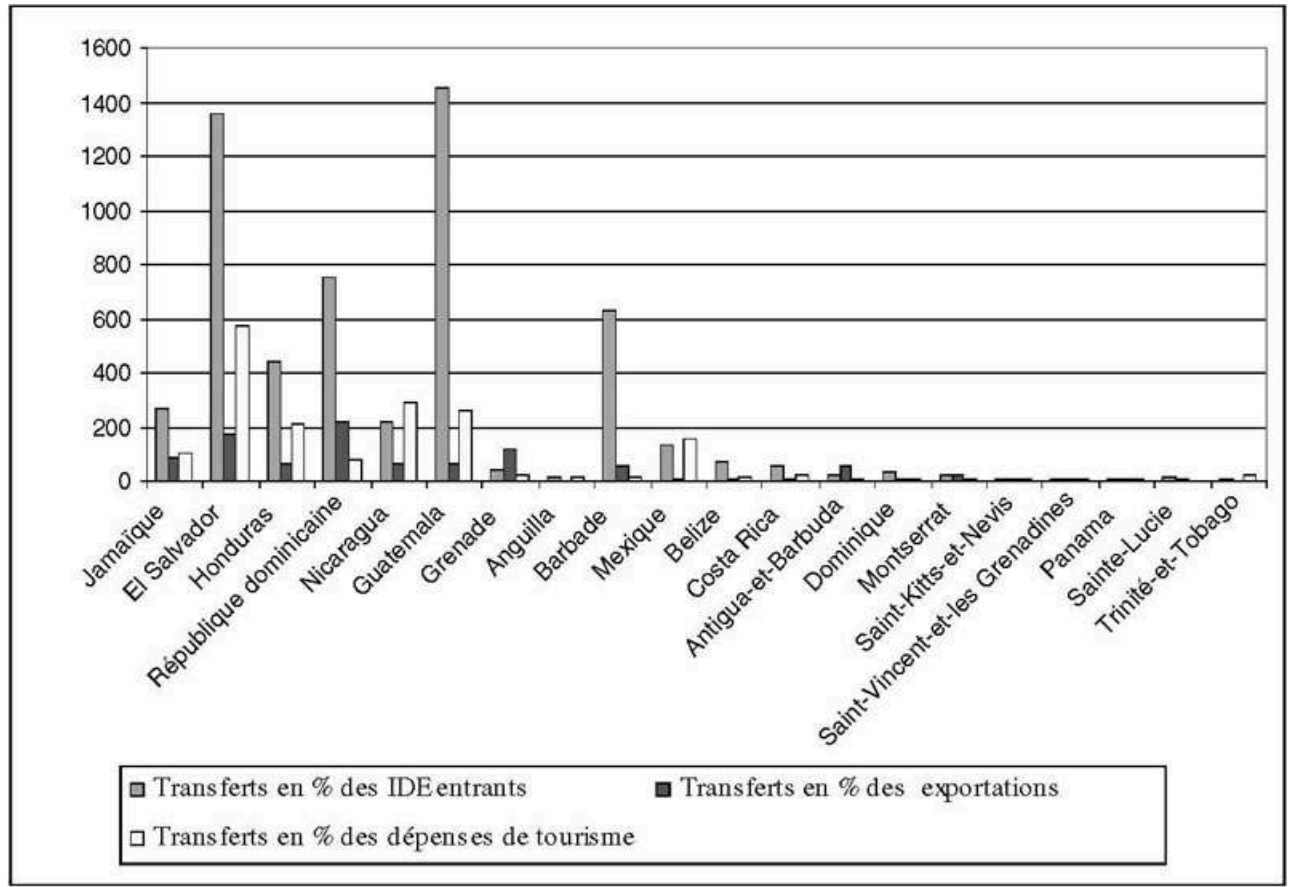

L'émigration des adultes le plus souvent masculins caractérise ces pays fournisseurs de main-d'œuvre internationale, dont les transferts jouent en quelque sorte un rôle de substitut aux investissements manquants. Les valeurs très élevées de l'indicateur des transferts en pourcentage des revenus du tourisme correspondent aux pays dans lesquels l'activité touristique est marginale, voire inexistante (Lesotho, Togo, et dans une moindre mesure Côte d'Ivoire). Enfin le Cap-Vert constitue un cas particulier, 
puisque les transferts y représentent près de sept fois ses exportations (J. Lourenço, C. Foy, 2003).

L'importance des transferts migratoires dans les économies d'Amérique latine (derrière celles d'Amérique centrale) est indéniable. Ainsi en Équateur les transferts sont la deuxième ressource financière du pays après le pétrole. Ils sont cinq fois supérieurs au crédit accordé par le FMI en 2001. Cependant d'autres pays sont très peu dépendant de ces transferts. C'est en particulier le cas du Chili, dont le développement économique fondé sur les exportations s'est confirmé depuis deux décennies, où ils ne représentent que $0,02 \%$ du son PIB en 2003, soit 0,79 \$ US par habitant.

En Afrique du Nord et au Moyen-Orient (figure 8), l'indicateur transferts en pourcentage des revenus du tourisme n'est pas approprié à toutes les situations, car cette activité ne joue qu'un rôle réduit. C'est le cas du Yémen, où les transferts des migrants représentent plus de 34 fois les revenus du tourisme, ce qui nous a amené, pour des raisons de commodité graphique, à ne pas faire figurer pour ce pays le rapport entre ces deux flux financiers.

Fig. 8. Afrique du Nord et Moyen Orient : transferts migratoires, investissements étrangers, exportations et tourisme, 2003

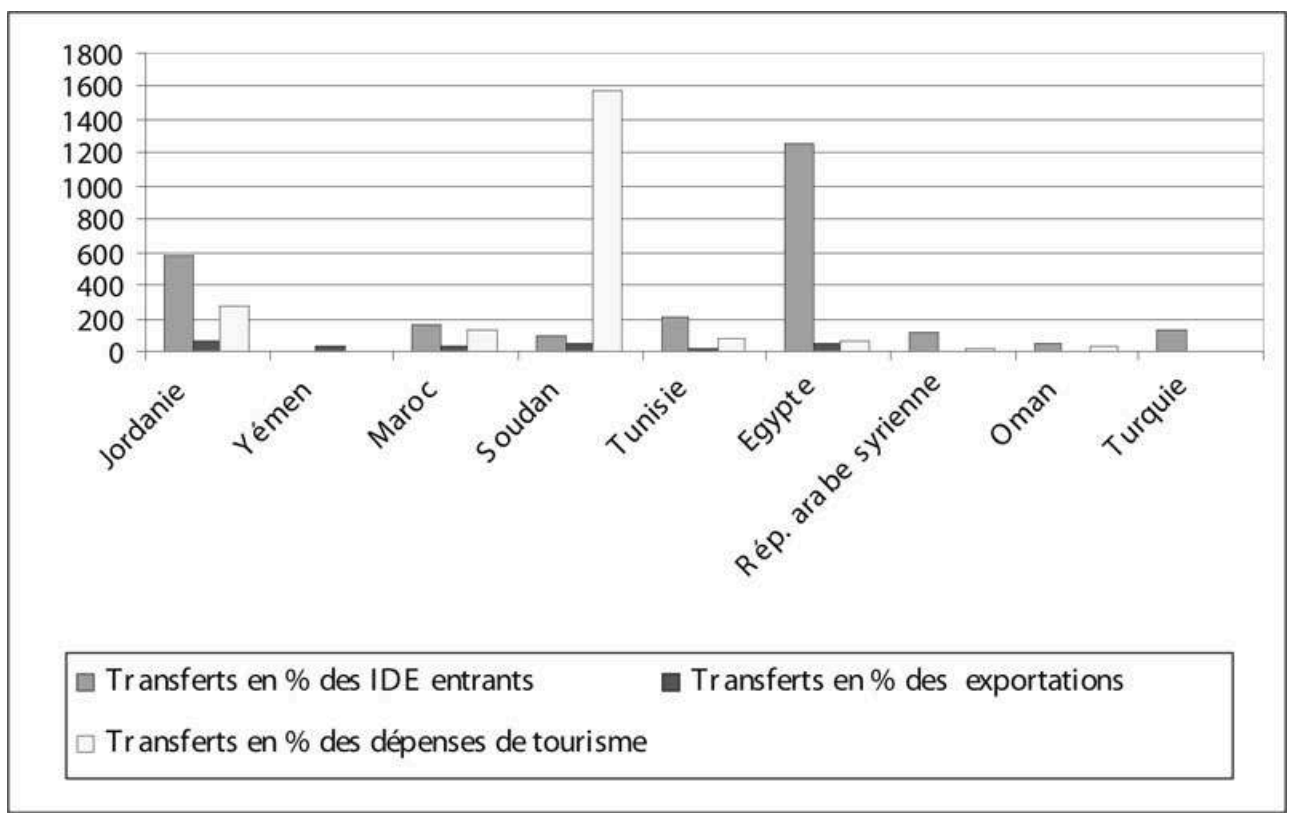

Dans la région, le pays le plus déficitaire en investissements étrangers relativement aux transferts des migrants est l'Égypte. Enfin il faut noter que bien qu'étudié ici dans le cadre des transferts entrants, Oman comme les autres pays producteurs de pétrole du Golfe, est un pays émetteur net de transferts. Les transferts entrants y ont donc une place marginale. 
Fig. 9. CEl et Europe du Sud-est : transferts migratoires, investissements étrangers, exportations et tourisme, 2003

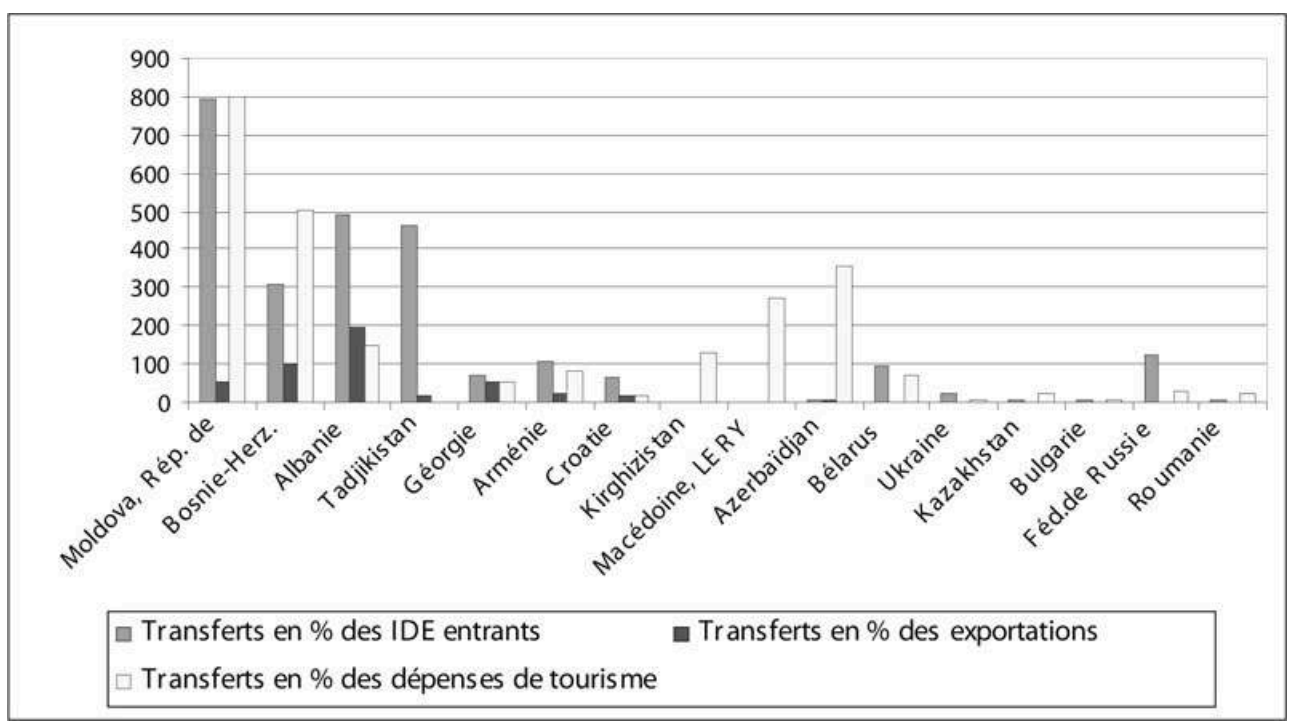

La plupart des pays de la CEI et de l'Europe du Sud-est bénéficient de peu d'investissements étrangers et de recettes touristiques faibles. Aussi les transferts des migrants peuvent être 5 à 8 fois plus élevés que ces deux autres entrées (Moldavie, Albanie, Azerbaïdjan, etc.). Au Kirghizistan, où les transferts migratoires sont quarante fois plus élevés que les revenus du tourisme; aussi avons-nous renoncé, pour des raisons de commodité graphique, à faire figurer pour ce pays le rapport entre ces deux flux financiers.

En revanche dans les pays producteurs de pétrole, comme le Kazakhstan par exemple, les transferts migratoires n'ont pas le même poids relatif, puisque la mise en valeur de l'or noir a impliqué des investissements lourds.

Le groupe de pays inclus dans la région « Asie et Océanie » (que nous avons construite en agrégeant les deux catégories Asie et Océanie employées par le Manuel de Statistiques de la CNUCED) pose un problème majeur d'hétérogénéité. Dans ce groupe se trouvent des pays aussi différents que le Bangladesh, pour lequel le PIB par habitant s'élève à un peu plus de $360 \$$ en 2002, et la Corée du Sud, qui est 30 fois plus riche avec un PIB par habitant se montant à 10000 \$. Aussi, à la différence des autres histogrammes, celui-ci est construit sur une échelle logarithmique, ce qui en rend la lecture moins immédiate. 
Fig. 10. Asie et Océanie : transferts migratoires, investissements étrangers, exportations et tourisme, 2003

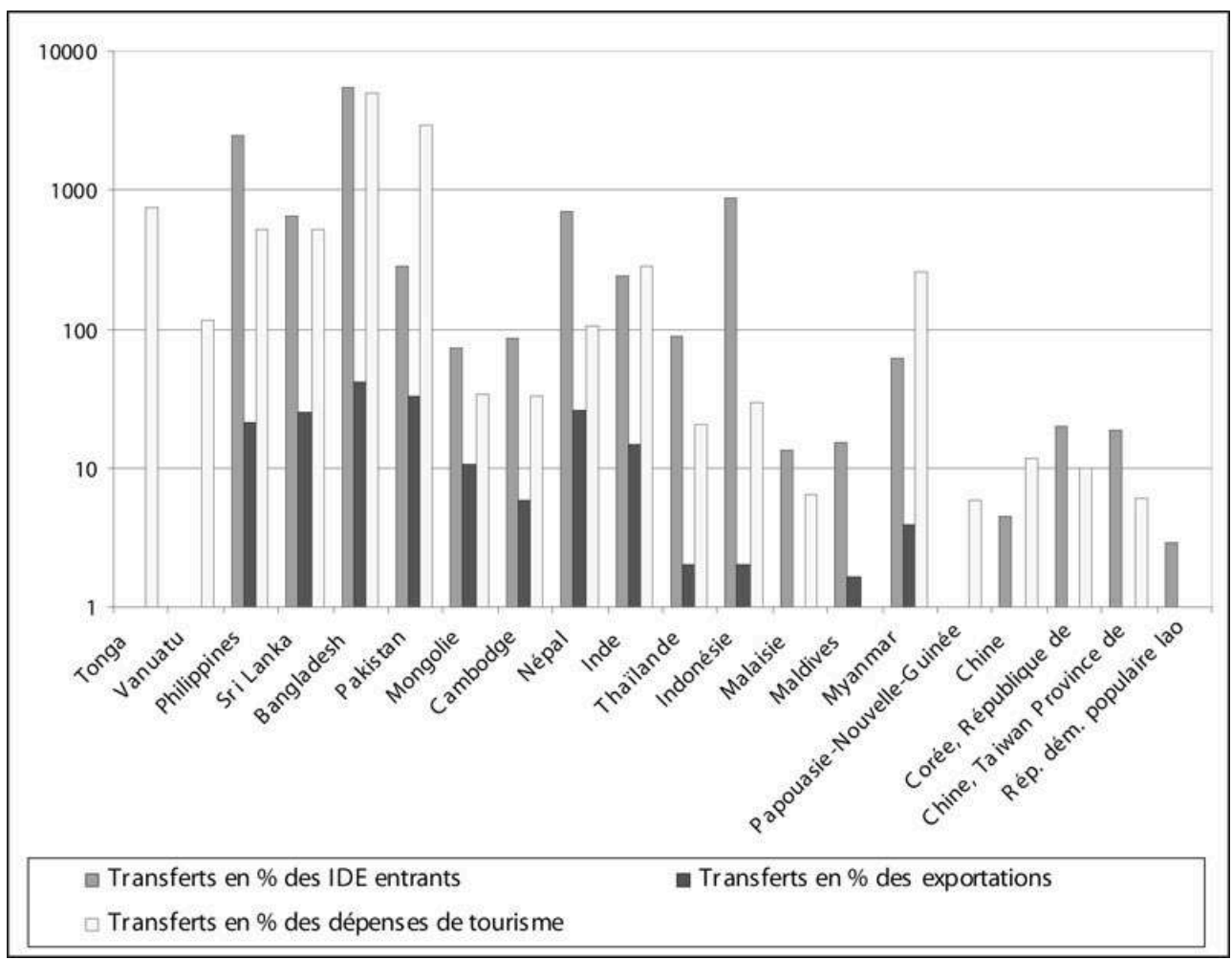

Les transferts sont particulièrement disproportionnés par rapport aux investissements étrangers au Bangladesh, avec un rapport de 55 à 1 en faveur des transferts. C'est aussi ce que l'on peut noter pour les Philippines ou l'Indonésie fournisseurs de main-d'œuvre aussi bien dans la région (Malaisie, Japon) que dans les économies pétrolières des pays du Golfe. Le déficit d'investissements privés dans ces pays est particulièrement accusé si l'on compare leur situation à celle de leurs « voisins » préférés des investisseurs, tels que la Corée ou Taïwan, dont le PIB par tête est par ailleurs bien supérieur, et que l'on ne s'étonne pas de trouver en queue d'échantillon (les pays sont classés en fonction décroissante de la part que représentent leurs transferts entrants dans le PIB).

\section{Conclusion : les limites de la connaissance des flux financiers}

$\mathrm{Au}$ terme de ce bref panorama sur les transferts migratoires, il faut d'abord rappeler que la question des transferts informels n'a pas été abordée. Mais on l'a dit, ce problème se pose surtout dans la perspective de la contribution des transferts au développement: comment en effet identifier la contribution des divers facteurs financiers qui contribuent à la spéculation immobilière, à l'amélioration des techniques agricoles, ou encore au développement des infrastructures communautaires si l'on ignore le poids respectif des transferts formels et informels? Il s'agissait simplement ici de comparer les flux de transferts officiels à deux autres postes de la balance des paiements.

Au-delà de l'objectif d'homogénéisation des données, qui semble atteint de façon satisfaisante avec la diffusion et la mise en application des définitions du FMI, 
l'amélioration de la qualité des données relatives aux transferts officiels devra passer dans un premier temps par l'augmentation et la rationalisation des moyens de collecte des données dans les pays en développement. Mais il faut bien noter qu'on touche là à la limite des méthodes statistiques: mieux cerner la nature et l'origine des flux impliquerait d'exiger plus d'informations des organismes producteurs de sources statistiques, c'est-à-dire en particulier des banques commerciales, exigence que cellesci répercuteraient sur leurs clients expéditeurs ou destinataires de fonds. Or une telle démarche risquerait d'accentuer la méfiance des populations à l'égard de pratiques perçues comme inquisitrices et d'inciter les expéditeurs à se tourner vers l'économie souterraine, substitut toujours à disposition. Rappelons en effet que les études convergent pour souligner la faible marge de manœuvre des États. Les mesures autoritaires de contrôle des changes (celles qui visent à défendre la monnaie nationale en la surévaluant de manière artificielle (CERED, 1996: 210, 253; Brown, 1994: 3), aboutissent quasi systématiquement à une explosion du marché noir, dans lequel les transferts des travailleurs, tout comme les autres capitaux, vont s'engouffrer ${ }^{11}$.

En un mot, en dépit de ces réserves, ces données sont les seules dont on dispose pour avoir une vue d'ensemble des transferts officiels au niveau mondial.

\section{BIBLIOGRAPHIE}

BANQUE MONDIALE International Technical Meeting on Measuring Migrant Remittances (January 24-25, 2005). Http //www.worldbank.org/data/remittances.html

NATIONS UNIES Fourth Coordination Meeting on International Migration, New York, 26-27 OCTOBER 2005

http //www.un.org/esa/population/publications/fourthcoord2005/fourthcoord2005.htm CNUCED, Manuel de Statistiques de la CNUCED

http : //www.unctad.org/Templates/Page.asp?intItemID=1890\&lang=1

OCDE (Données sur les migrations)

http : //www.oecd.org/document/36/0,2340,fr $2649374152917397311137415,00 . h t m l$

BENBOUZID Mohamed (1999) Réseaux financiers et marchés parallèles de devises : les Algériens dans l'économie informelle, Revue Européenne des Migrations Internationales, vol. 15, n 2, pp. 123-139.

BROWN Richard P.C. (1994) Consumption and investments from migrants' remittances in the South Pacific, International Migration Papers, ILO, Geneva.

CHARBIT Yves (2006) Transferts, retours et développement. Données, concepts, problématiques, in Véronique Petit éd., Les migrations internationales de retour dans la perspective des pays du Sud, Paris, CEPED, pp. 44-75. 
CHAREF Mohamed (2006) La rente financière marocaine : quelles stratégies pour quels enjeux?, in Véronique Petit Ed., Les migrations internationales de retour dans la perspective des pays du Sud, Paris, CEPED, pp. 76-104.

CFSI (2003) Migration et phénomènes migratoires, Rapport, Paris.

ELLERMAN David (2003) Policy Research on Migration and Development, World Bank Policy Research Working Paper 3117, 64 p.

HARRISON Anne (2004) Working Abroad - the benefits flowing from nationals working in other economies, Round Table on Sustainable Development, Paris, OECD, 80 p.

IÇDUYGU Ahmet (2005) International Migrants Remittances in Turkey, CARIM, Istanbul, 16 p.

LEBON André (1994) Situation de l'immigration et présence étrangère en France 1993-1994, Direction de la population et des migrations, Paris, $125 \mathrm{p}$.

LOPEZ ESPINOSA Mario Estudio sobre remesas de mexicanos en el exterior y su vinculación con el desarrollo económico, social y cultural de sus comunidades de origen, Estudios sobre Migraciones Internacionales, 59, Programa de Migraciones Internacionales, ILO, Genève, 2002.

LOURENÇO Jaime, FOY Colm (2003) Cap-Vert : gouvernance et développement, OCDE, Document de travail $n^{\circ} 225,60 p$.

LUNA MARTINEZ, José de (2005) Workers' Remittances to Developing Countries : A Survey with Central Banks on Selected Public Policy Issues, World Bank Policy Research Working Paper 3638, 43 p.

MBOUP Mourtala (2000) Les Sénégalais d'Italie, Paris, L'Harmattan.

RATHA Dilip, (2004) Understanding the Importance of Remittances, World Bank, 5 p.

SAFIR Nadji (1996) La problématique migratoire internationale algérienne dans les années 90 : vers de nouvelles perspectives, Migration internationale, CERED, Maroc, pp. 193-211.

SANDER Cerstin, MAIMBO Samuel M. (2003) Migrant Labor Remittances in Africa : Reducing Obstacles to Developmental Contributions, Africa Region Working Paper Series $n^{\circ}$ 64, $65 \mathrm{p}$.

SOLIMANO Andrés (2003) Remittances by immigrant, Issues and Evidence, UNU-WIDER, Discussion Paper $n^{\circ} 2003 / 89,25$ p.

\section{ANNEXES}


Tableau 5 : Transferts reçus dans 103 pays en développement en 1985 et 2003

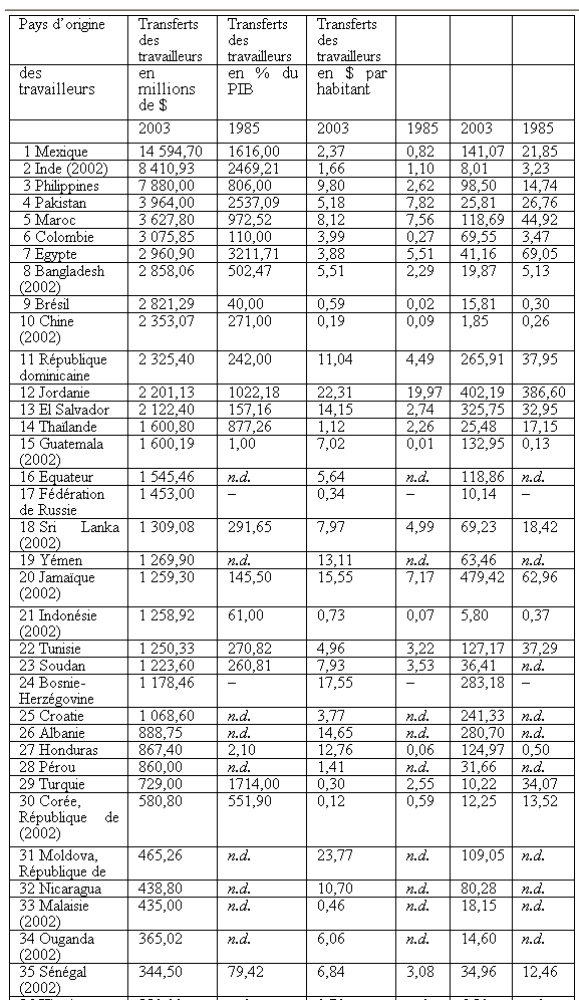

\section{Tableau 5 (suite)}

\begin{tabular}{|c|c|c|c|c|c|c|}
\hline 35 Sénégal & 344,50 & 79,42 & 6,84 & 3,08 & 34,96 & 12,46 \\
\hline Ukraine & 330,00 & n.d. & 0,70 & n.d. & 6,80 & \begin{tabular}{|l|} 
n.d. \\
.
\end{tabular} \\
\hline $\begin{array}{l}37 \text { Costa Rica } \\
\text { 38 Afrique du }\end{array}$ & $\begin{array}{l}320,88 \\
288,40\end{array}$ & $\frac{1,20}{38,71}$ & $\frac{1,84}{0,27}$ & $\frac{0,14}{0,07}$ & & $\frac{2,13}{1,24}$ \\
\hline 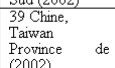 & 273,00 & n.d. & 0,10 & n.d. & 12,11 & n.d. \\
\hline (2002) Argentine & 252,85 & 27,00 & 0,19 & 0,03 & 6,58 & 0,89 \\
\hline $\begin{array}{l}41 \text { Géorgie } \\
2 \text { Paraguay }\end{array}$ & $\frac{245,74}{22210}$ & $\frac{n . d .}{0.60}$ & $\frac{6,23}{377}$ & $\begin{array}{ll}n . d_{1} \\
0.1\end{array}$ & 47,94 & n.d. \\
\hline $\begin{array}{l}42 \text { Paraguay } \\
43 \text { Lesotho }\end{array}$ & $\frac{222,10}{183,61}$ & $\frac{9,60}{223,92}$ & $\frac{3,71}{26,42}$ & $\begin{array}{l}0,21 \\
87,81\end{array}$ & $\frac{3,78}{102,01}$ & \begin{tabular}{|l}
2,66 \\
146,74
\end{tabular} \\
\hline (2014) Azerbaidjan & 170,96 & n.d. & 2,40 & n.d. & 20,42 & n.d. \\
\hline $\begin{array}{l}45 \text { Arménie } \\
46 \text { Bélanus }\end{array}$ & $\frac{165,70}{16170}$ & n.d. & $\frac{5,98}{092}$ & n.d. & $\frac{54,14}{1624}$ & n.d. \\
\hline $\begin{array}{l}\text { 46 Bélaurs } \\
77 \text { Kazakhstan }\end{array}$ & $\frac{161,70}{147,08}$ & $\frac{n . d .}{n . d .}$ & 0,92 & $\frac{n . d .}{n . d .}$ & $\frac{16,34}{9,53}$ & $\begin{array}{l}n . d . \\
n . d . \\
\end{array}$ \\
\hline $\begin{array}{l}48 \text { Népal } \\
\end{array}$ & 146,99 & n.d. & 2,69 & n.d. & 6,11 & n.d. \\
\hline 49 Tadjikistan & 145,98 & $n d$. & 9,39 & nd. & 23,37 & $n . d$. \\
\hline $\begin{array}{l}50 \text { Côte } \\
\text { d'Ivoire }\end{array}$ & 141,03 & 24,04 & 0,96 & 0,35 & 8,48 & 2,43 \\
\hline$\frac{51 \mathrm{Mali}}{20002)}$ & 137,65 & 67,00 & 4,37 & 5,79 & 10,91 & 9,07 \\
\hline \begin{tabular}{|l}
52 République \\
arabe syrienne \\
$(2002)$
\end{tabular} & 135,00 & 349,60 & 0,51 & 2,38 & 7,77 & 33,63 \\
\hline 53 Bolivie & 126,00 & 6,00 & 1,63 & 0,09 & 14,30 & 1,02 \\
\hline $\begin{array}{l}54 \text { Roumanie } \\
55 \text { Cambodge }\end{array}$ & $\begin{array}{l}124,00 \\
123,00\end{array}$ & $\frac{n . d .}{n . d .}$ & $\frac{0,22}{3,34}$ & $\frac{n . d .}{n . d .}$ & $\frac{5,55}{8,91}$ & \begin{tabular}{|l|}
$n . d$. \\
$n . d$.
\end{tabular} \\
\hline 56 Myanmar & 117,28 & n.d. & 0,23 & n.d. & 2,43 & n.d. \\
\hline 57 Barbade & 109,15 & 8,15 & 4,24 & 0,68 & 405,13 & 32,21 \\
\hline 8 Macédoine, & 105,90 & n.d. & 2,85 & n.d. & 51,77 & n.d. \\
\hline 59 Cap-Vert & 92,25 & 20,76 & 11,28 & 15,04 & 199,16 & 66,96 \\
\hline 60 Panama & 85,30 & 99,00 & 0,80 & 1,98 & 27,34 & 45,69 \\
\hline 61 Bénin & 83,60 & 40,29 & 3,53 & 3,85 & 13,09 & 9,96 \\
\hline & 69,48 & 40,80 & 1,66 & 0,67 & 3,68 & 3,01 \\
\hline 63 Togo $(2001)$ & 68,50 & 15,41 & 5,15 & 2,05 & 14,62 & 5,09 \\
\hline & 67,29 & $n . d$. & 0,34 & $\frac{n . d .}{n .07}$ & $\frac{8,52}{311}$ & $\begin{array}{l}n . d . \\
n .32 \\
\end{array}$ \\
\hline $\begin{array}{l}65 \text { Ghana } \\
66\end{array}$ & 61,75 & 47,55 & 5,30 & 12,99 & $\frac{3,11}{57,75}$ & $\frac{0,33}{n . d .}$ \\
\hline 67 Mongolie & 56,31 & n.d. & 5,04 & n.d. & 22,00 & n.d. \\
\hline$\frac{(2002)}{68}$ Vanuatu & 52,70 & 8,74 & 24,63 & 7,40 & 261,13 & n.d. \\
\hline 69 Tonga & 52,53 & 21,87 & 40,72 & 36,45 & 517,11 & n.d. \\
\hline 70 Guyana & 51,00 & n.d. & 6,85 & n.d. & 66,78 & n.d. \\
\hline
\end{tabular}


Tableau 5 (suite)

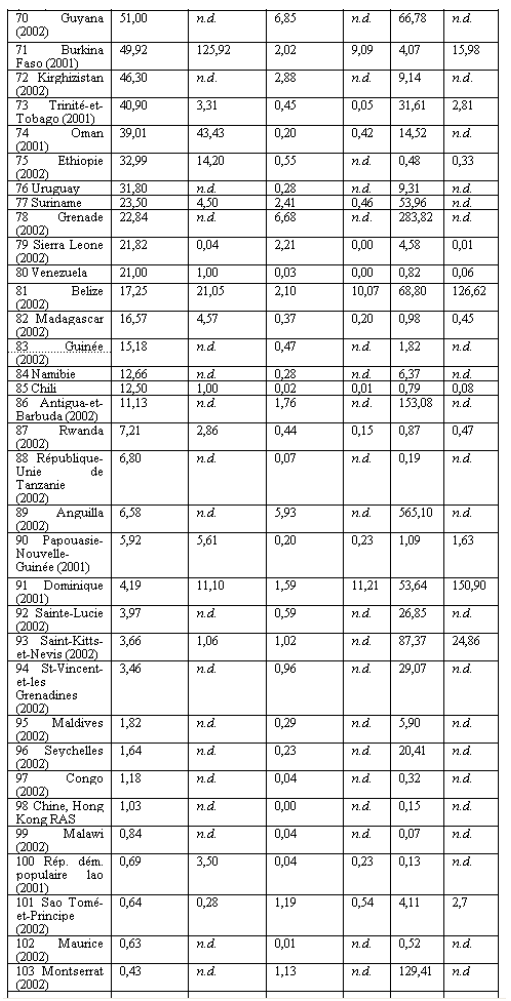

Source : Manuel de Statistiques de la CNUCED, 2005

Tableau 6 : Afrique sub-saharienne. Transferts des travailleurs, PIB, exportations, revenus du tourisme et investissements directs étrangers

\begin{tabular}{|c|c|c|c|c|c|}
\hline Pays & $\begin{array}{c}\text { Transferts en } \\
\text { millions de } \$ \\
\text { US }\end{array}$ & $\begin{array}{c}\text { Transferts } \\
\text { en \% du PIB }\end{array}$ & $\begin{array}{l}\text { Transferts en \% } \\
\text { des exportations }\end{array}$ & $\begin{array}{c}\text { Transferts en \% } \\
\text { des revenus du } \\
\text { tourisme }\end{array}$ & $\begin{array}{c}\text { Transferts } \\
\text { en \% des IDE } \\
\text { entrants }\end{array}$ \\
\hline Lesotho & *183,61 & $* 26,42$ & $* 38,49$ & $* 918,04$ & $* 675,03$ \\
\hline Cap-Vert & 92,25 & 11,28 & 678,32 & *128,91 & 667,89 \\
\hline Sénégal & $*_{344,50}$ & $*_{6,84}$ & $*_{25,89}$ & *190,33 & $*_{638,59}$ \\
\hline Ouganda & $*_{365,02}$ & $* 6,06$ & $* 64,91$ & *197,31 & $*_{146,40}$ \\
\hline Swaziland & $* 61,75$ & $*_{5,30}$ & $*_{5,37}$ & $*_{237,48}$ & $* 130,09$ \\
\hline Togo & $* * 68,50$ & $* * 5,15$ & $* * 16,06$ & $* * 622,75$ & $* * 96,75$ \\
\hline Mali & $*_{137,65}$ & $*_{4,37}$ & $* 14,80$ & nd & $* 135,13$ \\
\hline Bénin & $* * 83,60$ & $* * 3,53$ & $* * 18,65$ & $* * 114,52$ & $* * 201,59$ \\
\hline Sierra Leone & $*_{21,82}$ & $*_{2,21}$ & $* 23,65$ & $* 66,11$ & $*_{559,37}$ \\
\hline
\end{tabular}




\begin{tabular}{|c|c|c|c|c|c|}
\hline Burkina Faso & ** 49,92 & $* * 2,02$ & ** 21,06 & $* * 142,62$ & **609,83 \\
\hline Mozambique & 69,48 & 1,66 & 7,90 & $* 36,50$ & 20,64 \\
\hline $\begin{array}{l}\text { Sao Tomé-et- } \\
\text { Principe }\end{array}$ & $* 0,64$ & $* 1,19$ & $*_{9,72}$ & $* 6,45$ & $* 21,20$ \\
\hline Côte d'Ivoire & 141,03 & 0,96 & 2,41 & $* 240,18$ & 36,24 \\
\hline Ghana & 65,10 & 0,88 & 3,09 & $* 12,15$ & 47,66 \\
\hline Ethiopie & $* 32,99$ & $* 0,55$ & $*_{5,34}$ & $* 42,84$ & $* 43,98$ \\
\hline Guinée & $* 15,18$ & $* 0,47$ & $* 2,94$ & $*_{35,31}$ & $*_{50,61}$ \\
\hline Rwanda & $* 7,21$ & $* 0,44$ & $* 13,10$ & $* 23,24$ & $* 97,50$ \\
\hline Madagascar & $* 16,57$ & $* 0,37$ & $* 2,53$ & $* 46,04$ & *199,69 \\
\hline Namibie & 12,66 & 0,28 & 1,04 & $*_{3,68}$ & 15,16 \\
\hline Afrique du Sud & $* 288,40$ & $* 0,27$ & $* 0,79$ & $* 10,57$ & $* 38,11$ \\
\hline Seychelles & $* 1,64$ & $*_{0,23}$ & $*_{0} 0,59$ & $*_{1,27}$ & $*_{3,41}$ \\
\hline $\begin{array}{l}\text { République- } \\
\text { Unie de } \\
\text { Tanzanie }\end{array}$ & $* 6,80$ & $* 0,07$ & $* 0,56$ & $*_{0} 0,93$ & $* 2,83$ \\
\hline Malawi & $* 0,84$ & $* 0,04$ & $* 0,17$ & $*_{1}^{*}, 22$ & $*^{*} 14,30$ \\
\hline Congo & $* 1,18$ & $* 0,04$ & $* 0,05$ & $* 4,71$ & $* 0,77$ \\
\hline Maurice & $*_{0,63}$ & $*_{0,01}$ & $*_{0} 0,03$ & $*_{0,10}$ & $*_{1,94}$ \\
\hline
\end{tabular}

Notes : Données 2003, sauf : * Données 2002 ** Données 2001.

Source : Manuel de Statistiques de la CNUCED, 2005. 
Tableau 7 : Amérique latine. Transferts des travailleurs, PIB, exportations, revenus du tourismeet investissements directs étrangers

\begin{tabular}{|l|c|c|c|c|c|}
\hline Pays & $\begin{array}{c}\text { Transferts } \\
\text { en millions } \\
\text { de \$ US }\end{array}$ & $\begin{array}{c}\text { Transferts } \\
\text { en du PIB }\end{array}$ & $\begin{array}{c}\text { Transferts } \\
\text { en des } \\
\text { exportations }\end{array}$ & $\begin{array}{l}\text { Transferts } \\
\text { en des } \\
\text { revenus du } \\
\text { tourisme }\end{array}$ & $\begin{array}{l}\text { Transferts } \\
\text { en des } \\
\text { IDE entrants }\end{array}$ \\
\hline Guyana & $* 51,00$ & $* 6,85$ & $* 8,04$ & $* 104,08$ & $* 116,97$ \\
\hline Équateur & 1545,46 & 5,64 & 25,59 & 380,66 & 99,41 \\
\hline Colombie & 3075,85 & 3,99 & 24,27 & $* 257,79$ & 174,52 \\
\hline Paraguay & 222,10 & 3,77 & 14,94 & $* 325,00$ & 271,29 \\
\hline Suriname & 23,50 & 2,41 & 3,68 & $* 503,33$ & - \\
\hline Bolivie & 126,00 & 1,63 & 8,01 & 73,26 & 78,65 \\
\hline Pérou & 860,00 & 1,41 & 9,60 & 103,37 & 62,45 \\
\hline Brésil & 2821,29 & 0,59 & 3,86 & $* 78,49$ & 27,81 \\
\hline Uruguay & 31,80 & 0,28 & 1,45 & 10,00 & 12,09 \\
\hline Argentine & 252,85 & 0,19 & 0,86 & 12,41 & 52,90 \\
\hline Venezuela & 21,00 & 0,03 & 0,08 & 6,69 & 0,83 \\
\hline Chili & 12,50 & 0,02 & 0,06 & 1,45 & 0,42 \\
\hline
\end{tabular}

Notes : Données 2003, sauf : * Données 2002

Source : Manuel de Statistiques de la CNUCED, 2005.

Tableau 8 : Amérique centrale et Caraïbes. Transferts des travailleurs, PIB, exportations, revenus du tourisme et investissements directs étrangers

\begin{tabular}{|c|c|c|c|c|c|}
\hline \multirow[t]{2}{*}{ Pays } & \multirow{2}{*}{$\begin{array}{l}\text { Transferts } \\
\text { en millions } \\
\text { de \$ US }\end{array}$} & \multirow{2}{*}{$\begin{array}{l}\text { Transferts } \\
\text { en } \% \text { du PIB }\end{array}$} & Transferts & Transferts & Transferts \\
\hline & & & $\begin{array}{l}\text { en } \% \text { des } \\
\text { exportations }\end{array}$ & $\begin{array}{ll}\text { en } \% & \text { des } \\
\text { revenus du } & \text { dourisme }\end{array}$ & $\begin{array}{l}\text { en \% des } \\
\text { IDE entrants }\end{array}$ \\
\hline Jamaïque & $* 1259,30$ & $* 15,55$ & *90,07 & $* 104,16$ & $* 263,01$ \\
\hline $\begin{array}{c}\text { El } \\
\text { Salvador }\end{array}$ & 2122,40 & 14,15 & 169,12 & $* 571,26$ & 1356,17 \\
\hline Honduras & 867,40 & 12,76 & 65,10 & *209,97 & 438,08 \\
\hline $\begin{array}{l}\text { République } \\
\text { dominicaine }\end{array}$ & 2325,40 & 11,04 & 223,45 & 74,77 & 750,37 \\
\hline Nicaragua & 438,80 & 10,70 & 64,88 & 288,68 & 217,98 \\
\hline Guatemala & $* 1600,19$ & $* 7,02$ & $* 64,29$ & $* 261,47$ & *1452,08 \\
\hline Grenade & $* 22,84$ & $* 6,68$ & $* 115,61$ & $* 25,10$ & $* 39,65$ \\
\hline Anguilla & $* 6,58$ & $* 5,93$ & nd & $* 11,96$ & $* 18,01$ \\
\hline Barbade & ${ }^{*} 109,15$ & $* 4,24$ & $* 51,99$ & $* 16,84$ & *627,30 \\
\hline Mexique & 14594,70 & 2,37 & 8,82 & 154,33 & 135,34 \\
\hline Belize & $* 17,25$ & $* 2,10$ & $* 8,43$ & $* 12,97$ & $* 69,54$ \\
\hline Costa Rica & 320,88 & 1,84 & 5,26 & $* 23,25$ & 54,67 \\
\hline $\begin{array}{l}\text { Antigua-et- } \\
\text { Barbuda }\end{array}$ & $* 11,13$ & $* 1,76$ & $* 55,63$ & $* 4,06$ & $* 23,32$ \\
\hline Dominique & $* * 4,19$ & $* * 1,59$ & $* * 10,02$ & $* * 9,11$ & $* * 35,24$ \\
\hline Montserrat & ${ }^{*} 0,43$ & $* 1,13$ & $* 21,48$ & $* 4,77$ & $* 20,53$ \\
\hline $\begin{array}{c}\text { Saint- } \\
\text { Kitts-et-Nevis }\end{array}$ & $* 3,66$ & $* 1,02$ & $* 8,12$ & $* 6,53$ & $* 4,47$ \\
\hline $\begin{array}{l}\quad \text { St Vincent- } \\
\text { et-les } \\
\text { Grenadines }\end{array}$ & $* 3,46$ & $* 0,96$ & $* 9,08$ & $* 4,17$ & $* 10,66$ \\
\hline Panama & 85,30 & 0,80 & 9,87 & 10,60 & 10,78 \\
\hline $\begin{array}{l}\text { Sainte- } \\
\text { Lucie }\end{array}$ & $* 3,97$ & $* 0,59$ & $* 10,81$ & $* 1,89$ & $* 12,66$ \\
\hline $\begin{array}{l}\text { Trinité-et- } \\
\text { Tobago }\end{array}$ & $* * 40,90$ & $* * 0,45$ & $* * 1,05$ & $* * 20,35$ & $* * 4,90$ \\
\hline
\end{tabular}

Notes : Données 2003, sauf : * Données 2002 ** Données 2001.

Source : Manuel de Statistiques de la CNUCED, 2005 
Tableau 9 : Afrique du Nord et Moyen-Orient. Transferts des travailleurs, PIB, exportations, revenus du tourisme et investissements directs étrangers

\begin{tabular}{|c|c|c|c|c|c|}
\hline Pays & $\begin{array}{c}\text { Transferts } \\
\text { en millions } \\
\text { de \$ US }\end{array}$ & $\begin{array}{c}\text { Transferts } \\
\text { en } \% \text { du PIB }\end{array}$ & $\begin{array}{c}\text { Transferts } \\
\text { en des } \\
\text { exportations }\end{array}$ & $\begin{array}{l}\text { Transferts } \\
\text { en des } \\
\text { revenus du du } \\
\text { tourisme }\end{array}$ & $\begin{array}{l}\text { Transferts } \\
\text { en des } \\
\text { IDE entrants }\end{array}$ \\
\hline Jordanie & 2201,13 & 22,31 & 65,28 & 270,08 & 581,39 \\
\hline Yémen & 1269,90 & 13,11 & 33,14 & $* 3405,26$ & - \\
\hline Maroc & 3627,80 & 8,12 & 39,95 & 127,47 & 159,17 \\
\hline Soudan & 1223,60 & 7,93 & 44,09 & $* 1576,92$ & 90,69 \\
\hline Tunisie & 1250,33 & 4,96 & 15,58 & 84,77 & 214,13 \\
\hline Égypte & 2960,90 & 3,88 & 55,27 & 64,59 & 1247,22 \\
\hline $\begin{array}{c}\text { République } \\
\text { arabe } \\
\text { syrienne }\end{array}$ & $* 135,00$ & $* 0,51$ & $* 2,40$ & $* 9,48$ & $* 117,39$ \\
\hline Turquie & 729,00 & 0,30 & 1,57 & & \\
\hline Oman & $* * 39,01$ & $* * 0,20$ & $* * 0,35$ & $* * 26,90$ & $* * 46,87$ \\
\hline
\end{tabular}

Notes : Données 2003, sauf : * Données 2002 ** Données 2001.

Source : Manuel de Statistiques de la CNUCED, 2005

Tableau 10 : CEl et Europe du Sud. Transferts des travailleurs, PIB, exportations, revenus du tourisme et investissements directs étrangers

\begin{tabular}{|c|c|c|c|c|c|}
\hline Pays & $\begin{array}{r}\text { Transfert } \\
\text { s en } \\
\text { millions de } \\
\text { \$ US }\end{array}$ & $\begin{array}{r}\text { Transfert } \\
\text { s en \% du } \\
\text { PIB }\end{array}$ & $\begin{array}{r}\text { Transfert } \\
\text { s en \% des } \\
\text { exportations }\end{array}$ & $\begin{array}{r}\text { Transfert } \\
\text { s en \% des } \\
\text { revenus du } \\
\text { tourisme }\end{array}$ & $\begin{array}{r}\text { Transfert } \\
\text { en \% des } \\
\text { IDE } \\
\text { entrants }\end{array}$ \\
\hline $\begin{array}{c}\text { Moldova, } \\
\text { République de }\end{array}$ & 465,56 & 23,77 & 53,17 & 802,17 & 796,00 \\
\hline $\begin{array}{c}\text { Bosnie- } \\
\text { Herzégovine }\end{array}$ & 1178,46 & 17,55 & 102,47 & 503,61 & 309,38 \\
\hline Albanie & 888,75 & 14,65 & 196,37 & $* 150,63$ & 492,65 \\
\hline Tadjikistan & 145,98 & 9,39 & 17,54 & ${ }^{*} 3928,14$ & 461,22 \\
\hline Géorgie & 245,74 & 6,23 & 54,98 & ${ }^{*} 50,36$ & 72,78 \\
\hline Arménie & 165,70 & 5,98 & 24,44 & 80,44 & 106,63 \\
\hline Croatie & 1068,60 & 3,77 & 17,34 & 16,76 & 62,38 \\
\hline Kirghizistan & $* 46,30$ & $* 2,88$ & nd & $* 128,62$ & nd \\
\hline $\begin{array}{c}\text { Macédoine, } \\
\text { LERY }\end{array}$ & $* 105,90$ & $* 2,85$ & & $* 271,55$ & \\
\hline Azerbaïdjan & 170,96 & 2,40 & 6,60 & $* 356,29$ & 5,20 \\
\hline Bélarus & 161,70 & 0,92 & 1,62 & $* 72,69$ & 94,84 \\
\hline Ukraine & 330,00 & 0,70 & 1,43 & $* 6,99$ & 23,17 \\
\hline Kazakhstan & 147,08 & 0,53 & 1,14 & 26,08 & 7,11 \\
\hline $\begin{array}{c}* \text { Fédération } \\
\text { de Russie }\end{array}$ & 1453,00 & 0,34 & 1,08 & 32,27 & 127,01 \\
\hline Bulgarie & 67,29 & 0,34 & 0,89 & 4,15 & 4,74 \\
\hline Roumanie & 124,00 & 0,22 & 0,70 & $* 23,37$ & 7,92 \\
\hline
\end{tabular}

Notes : Données 2003, sauf : * Données 2002

Source : Manuel de Statistiques de la CNUCED, 2005 
Tableau 11 : Asie et Océanie. Transferts des travailleurs, PIB, exportations, revenus du tourisme et investissements directs étrangers

\begin{tabular}{|c|c|c|c|c|c|}
\hline \multirow[t]{2}{*}{ Pays } & Transferts & Transferts & Transferts & Transferts & \\
\hline & $\begin{array}{l}\text { en millions } \\
\text { de \$ US }\end{array}$ & en $\%$ du PIB & $\begin{array}{l}\text { en } \% \text { des } \\
\text { exportations }\end{array}$ & $\begin{array}{lr}\text { en } \% & \text { des } \\
\text { revenus du } \\
\text { tourisme }\end{array}$ & $\begin{array}{l}\text { en \% des } \\
\text { IDE entrants }\end{array}$ \\
\hline Tonga & $* * 52,53$ & $* * 40,72$ & nd & $* * 750,47$ & nd \\
\hline Vanuatu & $* * 52,70$ & $* * 24,63$ & nd & $* * 114,57$ & nd \\
\hline Philippines & 7880,00 & 9,80 & 21,59 & 517,40 & 2470,22 \\
\hline Sri Lanka & $* * 1309,08$ & $* * 7,97$ & $* 25,54$ & $* 517,42$ & $* 664,51$ \\
\hline Bangladesh & $* 2858,06$ & $* 5,51$ & $* 40,88$ & $* 5014,14$ & $* 5460,56$ \\
\hline Pakistan & 3964,00 & 5,18 & 33,23 & 2914,71 & 282,07 \\
\hline Mongolie & $* 56,31$ & $* 5,04$ & $* 10,64$ & $* 33,72$ & $* 72,38$ \\
\hline Cambodge & $* 123,00$ & $* 3,34$ & $* 5,84$ & $* 32,45$ & $* 84,78$ \\
\hline Népal & $* * 146,99$ & $* * 2,69$ & $* * 25,90$ & $* * 104,99$ & $* * 704,96$ \\
\hline Inde & $* 8410,93$ & $* 1,66$ & $* 15,02$ & $* 287,75$ & $* 243,87$ \\
\hline Thaïlande & 1600,80 & 1,12 & 1,99 & 20,47 & 88,83 \\
\hline Indonésie & $* 1258,92$ & ${ }^{*} 0,73$ & $* 2,01$ & $* 29,24$ & $* 868,22$ \\
\hline Malaisie & $* 435,00$ & $* 0,46$ & $* 0,44$ & $* 6,41$ & $* 13,58$ \\
\hline Maldives & $* 1,82$ & $* 0,29$ & $* 1,62$ & $* 0,57$ & $* 15,53$ \\
\hline Myanmar & $* * 117,28$ & $* * 0,23$ & $* * 3,85$ & $* * 260,63$ & $* * 61,08$ \\
\hline $\begin{array}{l}\text { Papouasie- } \\
\text { Nouvelle- } \\
\text { Guinée }\end{array}$ & $* * 5,92$ & $* * 0,20$ & nd & $* * 5,87$ & nd \\
\hline Chine & $* 2353,07$ & ${ }^{*} 0,19$ & ${ }^{*} 0,54$ & $* 11,54$ & $* 4,46$ \\
\hline $\begin{array}{c}\text { Corée, } \\
\text { République de }\end{array}$ & $* 580,80$ & ${ }^{*} 0,12$ & ${ }^{*} 0,30$ & $* 9,81$ & $* 19,75$ \\
\hline $\begin{array}{l}\text { Chine, } \\
\text { Taiwan } \\
\text { Province de }\end{array}$ & $* 273,00$ & ${ }^{*} 0,10$ & ${ }^{*} 0,19$ & $* 5,96$ & *18,89 \\
\hline $\begin{array}{l}\text { Rép. dém. } \\
\text { populaire lao }\end{array}$ & $* * 0,69$ & $* * 0,04$ & $* * 0,23$ & ${ }^{* * *} 0,66$ & $* * 2,89$ \\
\hline \begin{tabular}{l}
\multicolumn{2}{c}{ Chine, } \\
Hong Kong \\
RAS
\end{tabular} & 1,03 & 0,00 & 0,00 & nd & 0,01 \\
\hline
\end{tabular}

Notes : Données 2003, sauf : * Données 2002 ** Données 2001.

Source : Manuel de Statistiques de la CNUCED, 2005.

\section{NOTES}

1. Rappelons que les transferts monétaires officiels ne représentent qu'une partie de l'ensemble des flux : il faut en effet ajouter à ceux-ci les autres transferts monétaires, dits « informels », et ceux en nature, que l'on regroupe sous l'expression de « commerce à la valise ».

2. Technical Subgroup (TSG) on the Movement of Natural Persons - Mode 4

3. Pour avoir un ordre d'idée de l'importance de la composante informelle des transferts, on trouve par exemple dans l'ouvrage de Mario Lopez Espinosa (2002) des estimations de la part des transferts informels dans les transferts totaux de $20 \%$ pour le Bangladesh à $43 \%$ pour le Pakistan. D'autre part, nous nous intéressons ici aux seuls transferts internationaux. échappent donc à notre analyse les transferts internes ou domestiques, liés aux dynamiques migratoires internes à un pays. Ces dynamiques migratoires sont particulièrement importantes dans des pays à fortes disparités de développement régional comme la Chine.

4. Selon la distinction opérée parmi les migrants par les Nations Unies (Recommendations on Statistics of International Migration : Revision 1, United Nations, 1998)

5. La définition des Nations Unies prend garde d'exclure les personnes voyageant à l'étranger pour des motifs de tourisme, vacances, affaires, traitement médical, etc.

6. Sur le fonctionnement concret de ces transferts, voir Benbouzid (1999).

7. On notera que les transferts sortants ne représentent que $0,27 \%$ (tableau 2). Les entrants sont donc 2,4 fois supérieurs aux sortants. Ceci s'explique peut-être par la nature des transferts entrants en France, question qui sort des limites de cette étude. Enfin et surtout, ces deux 
pourcentages sont somme toute bien faibles, au regard de ceux relevés pour les pays en développement.

8. Voir en annexe les tableaux de données qui ont servi à construire les cartes.

9. Voir en particulier Sander et Maimbo, 2003.

10. Ainsi bien évidemment que tous les nouveaux états anciennement inclus dans l'URSS.

11. Trois exemples méritent d'être rappelés. Au Pakistan après l'essai nucléaire de mai 1998, le gouvernement introduisit un contrôle strict sur les comptes en devises. Tout retrait devait se faire en roupies au taux de 46 roupies pour 1 US\$ (contre 58 dans le système informel, dit hawala) : les transferts mensuels officiels passèrent de 150 millions de à 50 millions de dollars. Au Viêt-Nam, fin 1996, une taxe de $5 \%$ sur les transferts des migrants se traduisit par la diminution de $50 \%$ des flux (Buencamino et Gorbuvov, 2002 : 9-10 ; voir aussi Phong (2000: 195-198), sur l'inefficacité des mesures prises dans la période précédant le Doi moi de 1986). L'exemple a contrario des Philippines est remarquable : la remise à la parité du cours officiel et du cours sur le marché noir, en 1995, s'est traduite immédiatement par un quadruplement des transferts officiels. (Pour une expérience malheureuse en sens inverse entre 1983 et 1986, voir Brown, 1994 : 42)

\section{RÉSUMÉS}

Les transferts migratoires constituent un enjeu de taille pour les pays en développement. Nous analysons ici uniquement la part officielle des transferts, mesurée dans la Balance des Paiements, étant données les difficultés de mesure de leur composante informelle, dont le poids peut pourtant être lourd. Les données confirment l'hypothèse d'un taux de transfert plus élevé pour les migrants installés dans les pays du Golfe que dans les pays industrialisés à l'économie diversifiée tels la France. La présence des pays du Golfe dans le peloton de tête des pays émetteurs de transferts est la preuve du rôle des migrants dans la redistribution de la manne pétrolière. La comparaison des principales destinations des flux à près de vingt ans d'intervalle (1985 et 2003) pour un échantillon de 103 pays en développement, met en évidence une relative stabilité, du moins dans la hiérarchie des plus gros destinataires. Faut-il y voir une dépendance des pays à l'égard des flux de transferts? La comparaison des transferts à quatre indicateurs macro-économiques (PIB, revenus du tourisme, exportations, investissements directs étrangers (IDE) permet d'affiner l'analyse en révélant l'hétérogénéité intra-régionale des situations.

Migrants' remittances. Comparing industrial and developing countries. Remittances are a challenge for developing countries. Since it impossible to provide exact figures of informal transfers, although they are probably very large, only official money transfers are dealt with here, as one of the components of the balance of payments. Data confirm that migrants working in Gulf countries transfer a larger share of their income that those residing in diversified economies such as that of France. The high ranking of Gulf countries in the list of countries sending remittances prove that migrants play an important part in the redistribution of income generated by oil. A comparison based on a sample of 103 countries over a period of nearly twenty years (1985-2003) shows that those receiving large amount of remittances are more or less the same, which suggests a dependency of these countries on remittances. However the reality is more complex. Comparing remittances to four other macroeconomic indicators (GDP, tourism 
receipts, direct foreign investment, exports) within each region of the world, reveals large intraregional differences between countries.

Las transferencias monetarias de los inmigrantes: países industrializados y en desarrollo. Las transferencias migratorias, es decir los envíos de los emigrantes hacia sus países de origen, revisten gran importancia para los países en desarrollo. Este artículo analiza únicamente las transferencias oficiales contabilizadas por la Balanza de Pagos. La medida de las transferencias informales resulta en efecto demasiado difícil para integrarla en un estudio estadístico, a pesar de que su peso puede ser importante. Los datos confirman la hipótesis de una tasa de transferencia más elevada en el caso de los inmigrantes instalados en los países del Golfo que en el de sus homólogos instalados en países industrializados con una economía diversificada como Francia. La presencia de los países del Golfo en el pelotón de cabeza de los países emisores de transferencias es la prueba del rol que los inmigrantes juegan en la distribución de las ganancias petroleras. Sobre la base de una muestra de 103 países en desarrollo, la comparación de las principales destinaciones de los flujos de los años 1985 y 2003 evidencia una estabilidad relativa, al menos en la jerarquía de los mayores destinatarios. ¿Se debe ver en ello una dependencia de los países respecto a los flujos financieros? La comparación de las transferencias con cuatro indicadores macro-económicos (PIB, recetas turísticas, exportaciones, inversiones directas extranjeras IDE) permite afinar el análisis revelando la heterogeneidad intraregional de las situaciones.

\section{INDEX}

Mots-clés : développement économique, relations avec le pays d'origine, relations nord-sud, statistiques, transfert de fonds

\section{AUTEURS}

\section{YVES CHARBIT}

Professeur de démographie, Université Paris Descartes, Laboratoire POPINTER, 45 rue des Saints Pères, 75006 Paris, France. Courriel : yves.charbit@paris5.sorbonne.fr

\section{ISABELLE CHORT}

École Normale Supérieure, 45 rue d'Ulm, Chercheur associé à POPINTER. Courriel : isabelle.chort@ens.fr 